\title{
Causal Links between Dorsal Medial Superior Temporal Area Neurons and Multisensory Heading Perception
}

\author{
Yong Gu, ${ }^{1,2}$ Gregory C. DeAngelis, ${ }^{3}$ and Dora E. Angelaki ${ }^{1,4}$ \\ ${ }^{1}$ Department of Anatomy and Neurobiology, Washington University School of Medicine, St. Louis, Missouri 63110, ${ }^{2}$ Institute of Neuroscience, Chinese \\ Academy of Sciences, Shanghai 200031, China, ${ }^{3}$ Department of Brain and Cognitive Sciences, University of Rochester, Rochester, New York 14627, and \\ ${ }^{4}$ Department of Neuroscience, Baylor college of Medicine, Houston, Texas 77030
}

The dorsal medial superior temporal area (MSTd) in the extrastriate visual cortex is thought to play an important role in heading perception because neurons in this area are tuned to both optic flow and vestibular signals. MSTd neurons also show significant correlations with perceptual judgments during a fine heading direction discrimination task. To test for a causal link with heading perception, we used microstimulation and reversible inactivation techniques to artificially perturb MSTd activity while monitoring behavioral performance. Electrical microstimulation significantly biased monkeys' heading percepts based on optic flow, but did not significantly impact vestibular heading judgments. The latter result may be due to the fact that vestibular heading preferences in MSTd are more weakly clustered than visual preferences and multiunit tuning for vestibular stimuli is weak. Reversible chemical inactivation, however, increased behavioral thresholds when heading judgments were based on either optic flow or vestibular cues, although the magnitude of the effects was substantially stronger for optic flow. Behavioral deficits in a combined visual/vestibular stimulus condition were intermediate between the single-cue effects. Despite deficits in discrimination thresholds, animals were able to combine visual and vestibular cues near optimally, even after large bilateral muscimol injections into MSTd. Simulations show that the overall pattern of results following inactivation is consistent with a mixture of contributions from MSTd and other areas with vestibular-dominant tuning for heading. Our results support a causal link between MSTd neurons and multisensory heading perception but suggest that other multisensory brain areas also contribute.

\section{Introduction}

Precise and accurate heading estimation is crucial for navigation. Human and nonhuman primates can rely on either visual (optic flow) or vestibular (inertial motion) cues to discriminate heading angles as small as $1-2^{\circ}$ away from straight forward (Warren et al., 1988; Royden et al., 1992; van den Berg, 1992, 1996; Crowell and Banks, 1993; Britten and van Wezel, 1998, 2002; Gu et al., 2007, 2008, 2010; Fetsch et al., 2009; Butler et al., 2010). Moreover, human and nonhuman primates can integrate visual and vestibular cues in a statistically near-optimal fashion to improve precision, such that they can discriminate smaller headings when both cues are presented (Gu et al., 2008; Fetsch et al., 2009, 2011; Butler et al., 2010; de Winkel et al., 2010; Edwards et al., 2010).

Studies in macaques have provided evidence for convergence of optic flow and vestibular signals onto single neurons in multiple cortical areas, including the ventral intraparietal area (Colby et al., 1993; Bremmer et al., 2002a,b; Schlack et al., 2002, 2005; Chen et al., 2011c), the visual posterior sylvian area (Chen et al., 2011b), and the frontal pursuit area (Gu et al., 2010). However,

\footnotetext{
Received Oct. 12, 2011; revised Dec. 4, 2011; accepted Dec. 10, 2011.

Author contributions: Y.G., G.C.D., and D.E.A. designed research; Y.G. performed research; Y.G. analyzed data; Y.G., G.C.D., and D.E.A. wrote the paper.

This work was supported by NIH Grants EY019087 (D.E.A.) and EY016178 (G.C.D.).

Correspondence should be addressed to Dr. Dora E. Angelaki, Department of Neuroscience, Room S740, MS: BCM295, Baylor College of Medicine, One Baylor Plaza, Houston, TX 77030. E-mail: angelaki@bcm.edu.

DOI:10.1523/JNEUROSCI.5154-11.2012

Copyright $\odot 2012$ the authors $\quad 0270-6474 / 12 / 322299-15 \$ 15.00 / 0$
}

the most thoroughly studied area is the dorsal medial superior temporal area (MSTd) (Britten, 2008; Angelaki et al., 2009; Fetsch et al., 2010). Neurons in MSTd are tuned to complex patterns of optic flow that typically result from self-motion (Tanaka et al., 1986, 1989; Duffy and Wurtz, 1991, 1995), as well as to translation of the head/body in darkness (Duffy, 1998; Bremmer et al., 1999; Page and Duffy, 2003; Gu et al., 2006; Takahashi et al., 2007). Consistent with a role of MSTd in multisensory heading perception, trial-by-trial fluctuations in firing rates are correlated with monkeys' perceptual reports in a heading discrimination task (Gu et al., 2007, 2008). In addition, electrical microstimulation of MSTd biases heading perception based on optic flow (Britten and van Wezel, 1998, 2002), although a causal role of vestibular signals has not been tested.

To examine the causal role of area MSTd in multisensory heading perception, we manipulated neural activity using both electrical microstimulation and reversible chemical inactivation while animals performed a fine heading discrimination task. Our results confirm a causal link between MSTd and visual heading perception (Britten and van Wezel, 1998, 2002), and we establish, for the first time, a causal role of MSTd in vestibular heading judgments. Notably, effects of microstimulation and inactivation were considerably larger for visual than vestibular stimulation, consistent with visual dominance of heading tuning in MSTd. Importantly, even after inactivation of a large portion of MSTd bilaterally, animals could still combine cues near-optimally and reweight cues according to reliability. Using simple simulations, 
we show that our overall pattern of results is consistent with scenarios in which MSTd contributes to heading perception in conjunction with other areas that contain representations of heading that are dominated by vestibular input.

\section{Materials and Methods}

Motion stimuli. Three adult rhesus monkeys (Macaca mulatta) weighing $\sim 7 \mathrm{~kg}$ were trained using a custom-built virtual reality system. Translation of the monkey in the horizontal plane was accomplished by a motion platform (MOOG 6DOF2000E; Moog). To activate vestibular otolith organs, each transient inertial motion stimulus followed a smooth trajectory with a Gaussian velocity profile having a peak velocity of $4.5 \mathrm{~m} / \mathrm{s}$ (see Fig. $1 B$, "heading stimulus") and a peak acceleration of $\sim 1 \mathrm{~m} / \mathrm{s}^{2}$. This platform provided the inertial movements used in the "vestibular" and "combined" stimulus conditions.

A three-chip DLP projector (Christie Digital Mirage 2000) was mounted on the motion platform and rear-projected images (subtending $90 \times 90^{\circ}$ of visual angle) onto a tangent screen in front of the monkey. Visual stimuli depicted movement through a 3D cloud of "stars" that occupied a virtual space $100 \mathrm{~cm}$ wide, $100 \mathrm{~cm}$ tall, and $50 \mathrm{~cm}$ deep. Star density was $0.01 / \mathrm{cm}^{3}$, with each star being a $0.15 \times$ $0.15 \mathrm{~cm}$ triangle. Stimuli were presented stereoscopically as red/green anaglyphs and were viewed through Kodak Wratten filters (red no. 29; green no. 61). The display contained a variety of depth cues, including horizontal disparity, motion parallax, and size information. This system provided the optic flow stimuli used in the "visual" and "combined" stimulus conditions.

The motion coherence of the visual display was manipulated by randomizing the 3D location of a certain percentage of the stars on each display update while the remaining stars moved according to the specified heading ( $\mathrm{Gu}$ et al., 2008). This manipulation degrades optic flow as a heading cue and was used to reduce psychophysical sensitivity in the visual condition such that it roughly matched vestibular sensitivity.

Behavioral task. Monkeys were trained to perform a heading discrimination task around psychophysical threshold (see Fig. $1 \mathrm{~A}$ ). In each trial, the monkey experienced forward motion with a small leftward or rightward component (Gu et al., 2008). Monkeys were required to maintain fixation on a head-fixed visual target located at the center of the display screen during the motion stimulus. Trials were aborted if conjugate eye position deviated from a $2 \times 2^{\circ}$ electronic window around the fixation point. At the end of the $2 \mathrm{~s}$ trial, the fixation spot disappeared, two choice targets appeared to the left and right of the fixation spot, and the monkey made a saccade to one of the two targets to report his perceived motion as leftward or rightward relative to straight ahead. Across trials, heading was varied in fine steps around straight ahead (animal C: $\pm 9, \pm 2.7, \pm 0.81$, $\pm 0.24,0^{\circ}$, or $\pm 6, \pm 1.8, \pm 1, \pm 0.54, \pm 0.16,0^{\circ}$; animal S: $\pm 16, \pm 6.4$, $\pm 3.5, \pm 2.6, \pm 1,0^{\circ}$; animal $\left.\mathrm{J}: \pm 8, \pm 4, \pm 2, \pm 1, \pm 0.5,0^{\circ}\right)$. The range of headings, which was informed by extensive preliminary psychophysical testing using a staircase procedure, was adjusted to be fine enough to reliably measure thresholds yet broad enough to reliably estimate changes in sensitivity or bias associated with inactivation and microstimulation, respectively. metric function.

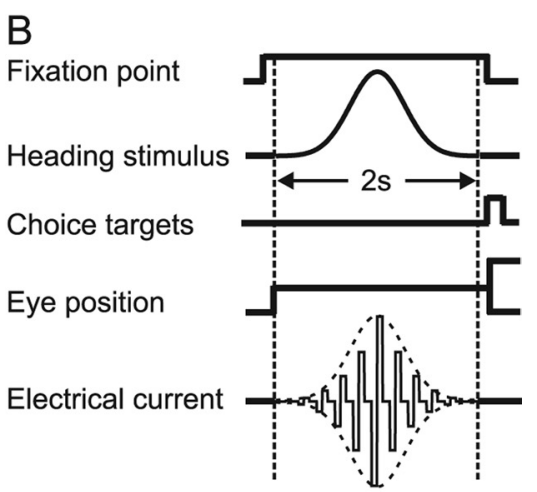

Inactivation

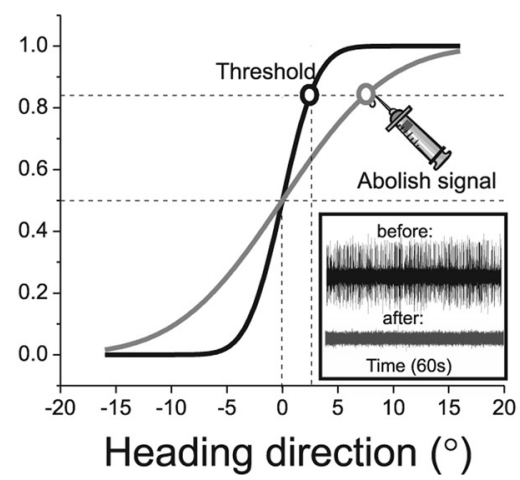

Figure 1. Heading discrimination task and hypothesized changes in behavioral performance. $\boldsymbol{A}$, Monkeys seated on a motion platform were translated forward, and heading angle, $\alpha$, was varied around straight ahead. A projector mounted on the platform displayed images of a 3D star field and provided optic flow cues. $\boldsymbol{B}$, Timing of events in the electrical microstimulation experiment. Afsual target, the monkey experienced forward motion with a small leftward or rightward component, and . Predicted effects of reversible inactivation on heading discrimination performance. Muscimol injection suppresses neural activity (inset) in a large region and is expected to deteriorate the precision of heading discrimination, leading to a shallower psycho-

The experimental paradigm consisted of three randomly interleaved stimulus conditions: (1) In the vestibular condition, the monkey was translated by the motion platform while fixating a head-fixed target on a blank screen. Thus, the main source of heading information was otolithdriven signals from the vestibular system (Gu et al., 2007), although we cannot exclude some contribution from other inputs, such as somatosensory or proprioceptive cues. (2) In the visual condition, the motion platform remained stationary while optic flow simulated the same range of headings. Visual motion coherence was adjusted for each monkey to match the psychophysical threshold measured for the vestibular condition (Gu et al., 2008). (3) In the combined condition, congruent inertial motion and optic flow were provided, with visual and vestibular stimuli being temporally synchronized (for more details, see $\mathrm{Gu}$ et al., 2006). Each stimulus condition was typically repeated a minimum of 10 times, but more typically $\sim 30$ times.

Experimental procedures. MSTd was identified through a combination of structural MRI and mapping of physiological response properties, such as direction selectivity for visual motion and large visual receptive fields encompassing the contralateral visual field and often including the fovea (for more details, see Gu et al., 2006).

In the electrical microstimulation experiments, we first recorded multiunit (MU) activity as the microelectrode (FHC; tip diameter, $\sim 3 \mu \mathrm{m}$; impedance, $\sim 500 \mathrm{k} \Omega$ ) was lowered into MSTd. We measured heading tuning in the horizontal plane (10 directions relative to straight ahead: 0 , 

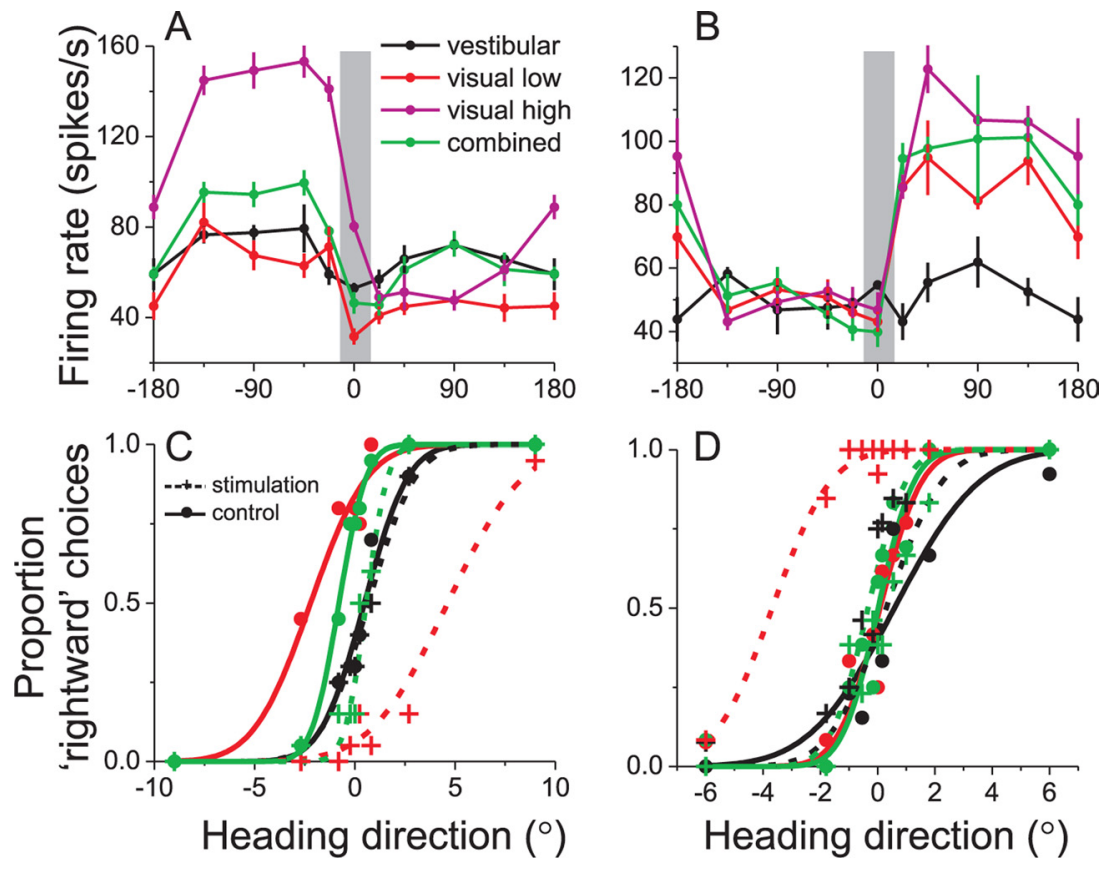

Figure 2. Examples of two microstimulation experiments. $\boldsymbol{A}, \boldsymbol{B}$, Heading tuning of MU activity at each stimulation site in response to vestibular (black), visual (low coherence, red; high coherence, magenta) and combined (green) stimuli. Error bars indicate SEM. Gray highlight, Heading range tested in the discrimination task. C, D, Psychometric functions for stimulated (dashed curves) and nonstimulated (solid curves) trials in response to vestibular (black), low-coherence visual (red), and combined (green) stimuli.
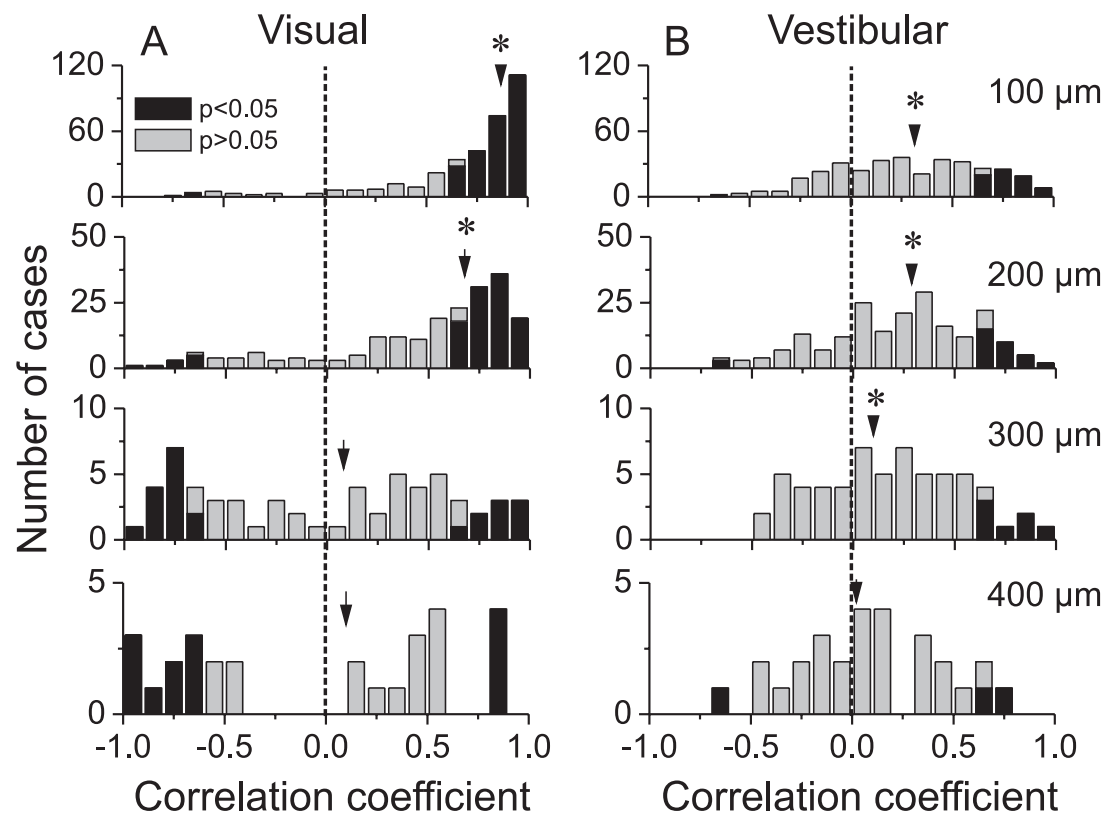

Figure 3. Clustering of visual $(\boldsymbol{A})$ and vestibular $(\boldsymbol{B})$ MU activity in area MSTd. Distributions of correlation coefficients of MU tuning across sites at different distances along an electrode penetration. The filled bars illustrate correlation coefficients significantly different from zero. The arrows illustrate median correlation values, and the asterisks mark cases for which the mean correlation coefficient is significantly different from zero ( $p<0.05$, sign test). The dashed vertical lines denote zero correlation.

$\pm 22.5, \pm 45, \pm 90, \pm 135$, and $\left.180^{\circ}\right)$ under both the vestibular and visual stimulus conditions (100\% motion coherence). For this measurement, monkeys were simply required to fixate a head-centered target while four to five repetitions were collected for each stimulus. Along each electrode penetration through MSTd, heading tuning curves were measured from MU activity for at least three to five consecutive recording sites, $100 \mu \mathrm{m}$ apart. We examined the sequence of heading tuning curves to identify regions in which at least two to three consecutive recording sites showed clear tuning with similar heading preferences in either the vestibular or the visual condition. A location in the midst of such a region was then chosen as the site for electrical stimulation, and the electrode was positioned at that location. Before microstimulation, MU tuning at the chosen site was reassessed using vestibular stimuli, low coherence visual stimuli (identical with those used in the discrimination task), and combined visual/vestibular stimuli. Since it was less common to find MU sites with significant and consistent vestibular heading tuning, priority was given to stimulating such sites even if the corresponding visual heading tuning was not so robust or consistent. Note that any biases introduced by this selection process should have favored finding microstimulation effects in the vestibular condition, which is opposite to what we observed.

Once a site was identified, low-amplitude electrical stimulation was delivered through the tip of the tungsten microelectrode while the monkey performed the fine heading discrimination task. Microstimulation consisted of $200 \mathrm{~Hz}$ pulse trains (biphasic, cathodal-anodal; pulse width, $200 \mu$ s for each phase duration; interpulse interval, $100 \mu \mathrm{s}$ ) that were $2 \mathrm{~s}$ in duration, coincident with the heading stimuli. The pulse train was amplitude modulated by a Gaussian envelope with a peak amplitude of $20 \mu \mathrm{A}$ (see Fig. $1 B$, "electrical current"), such that the envelope of the pulse train matched the velocity profile of the visual/ vestibular motion stimulus. This design was adopted to roughly match the temporal profile of microstimulation-induced neural activity with the average temporal profile of population activity in MSTd, which roughly follows stimulus velocity for both the visual and vestibular conditions (Gu et al., 2006; Chen et al., 2011a). Microstimulation was applied randomly during one-half of the trials in each experimental session. Experiments were performed daily, with one site generally stimulated in each experimental session.

In the reversible chemical inactivation experiments, the $\mathrm{GABA}_{\mathrm{A}}$ agonist muscimol (concentration, $10 \mathrm{mg} / \mathrm{ml}$ ) was injected using a "microinjectrode," which consists of a fine tungsten electrode inside of a thin cannula (Chen et al., 2001; Chowdhury and DeAngelis, 2008). The advantage of this approach is that it allows us to monitor MU activity before and after muscimol injection, such that silencing of neural activity can be confirmed before behavioral testing. This potentially reduces variability in the results that would be associated with failures to deliver the drug effectively (due to leaks, blockages, etc.). Briefly, the microinjectrode contains a fine tungsten electrode (FHC; tip diameter, $\sim 3 \mu \mathrm{m}$; body diameter, $75 \mu \mathrm{m}$; impedance, $\sim 500 \mathrm{k} \Omega$ ) that is inserted into a 32 $\mathrm{G}$ cannula. The end of the cannula mates to a three-port, T-shaped tubing connector. The electrode passes through the port opposite to where the cannula connects, is sealed to prevent leakage, and connects to the preamplifier for recording neural activity. The third port of the connector is attached via tubing to a Hamilton syringe filled with $10 \mathrm{mg} / \mathrm{ml}$ muscimol (Sigma-Aldrich). Ejection of drug from the Hamilton syringe 
was precisely controlled using a Harvard minipump. Before inserting the injectrode into the brain (through a guide tube), we first retracted a known volume of drug (typically $2 \mu \mathrm{l}$ ) from inside the injectrode assembly using the minipump. This prevented the drug from diffusing into the tissue during penetration, as a result of capillary action.

MU responses were monitored and response properties were mapped while the injectrode was lowered into the brain. Once the electrode tip (which typically extended $\sim 300-500 \mu \mathrm{m}$ beyond the end of the cannula) entered area MSTd, it was advanced to a depth of $\sim 700-$ $1000 \mu \mathrm{m}$ below the surface of MSTd, such that the tip of the cannula was roughly centered in the thickness of the cortex. This helped ensure that the drug spread roughly uniformly to the upper and lower layers of gray matter. Once the injectrode was situated in MSTd, we drove MU activity at the recording site by presenting fullfield optic flow in the preferred direction of the MU cluster. We then ejected the known volume of muscimol $(\sim 2 \mu \mathrm{l})$ needed to fill the injectrode. Subsequently, an additional $\sim 2 \mu \mathrm{l}$ of muscimol was injected very slowly $(0.1 \mu \mathrm{l} /$ $\min$ ) over $\sim 20 \mathrm{~min}$ to spread into the gray matter. This very slow injection helped ensure that pressure did not force the drug back up along the path of the cannula (Chowdhury and DeAngelis, 2008). Neural activity was typically silenced by the end of the $20 \mathrm{~min}$ injection period (see Fig. $1 D$ ), indicating successful delivery of the drug. In a few control experiments, we observed that neuronal activity was suppressed typically within a range of roughly 500-1000 $\mu \mathrm{m}$ below and above the recording site after the injection of $\sim 2 \mu \mathrm{l}$ of muscimol.

Given that receptive fields are large in MSTd and that retinotopy is coarse at best, we explored different spatial configurations of injection sites. Thus, we injected muscimol at one, two, or four sites in each experiment. Two-site injections were either applied to different hemispheres or they were applied to the same hemisphere at locations separated by $2-4 \mathrm{~mm}$ within the horizontal (transverse) plane. In experiments with injections at four sites, two injections were always made in each hemisphere, separated by $2-4 \mathrm{~mm}$ in the horizontal plane. Inactivation experiments were conducted on a weekly schedule. Data collected on the first $2 \mathrm{~d}$ (Monday, Tuesday) of each week served as controls ("Pre," preinjection). Muscimol injections were then made on Wednesday evenings and a brief session of behavioral data collection followed the injection ("0 h"). Extensive behavioral data were then collected on Thursday mornings (" $12 \mathrm{~h}$ ") and Friday mornings ("36 h"). Consistent with previous studies in MT using similar concentrations of muscimol, the effects of the drug generally disappeared between the 12 and $36 \mathrm{~h}$ time points. In three control experiments, we injected a physiological saline solution instead of muscimol, to confirm that there were no behavioral effects associated with the procedure in the absence of the drug. All other aspects of these control experiments were identical with those involving muscimol.

Data analysis and task details. Two types of analyses were performed in these experiments. First, we characterized MU activity by measuring mean firing rates during the middle $1 \mathrm{~s}$ of the $2 \mathrm{~s}$ stimulus period (where most of the motion occurs). These values were then used to generate tuning curves of MU activity, which were measured every $100 \mu \mathrm{m}$ along electrode penetrations in microstimulation experiments. The similarity between tuning curves recorded at different locations along a penetration was quantified using Pearson's correlation coefficients. In addition, the
Monkey C

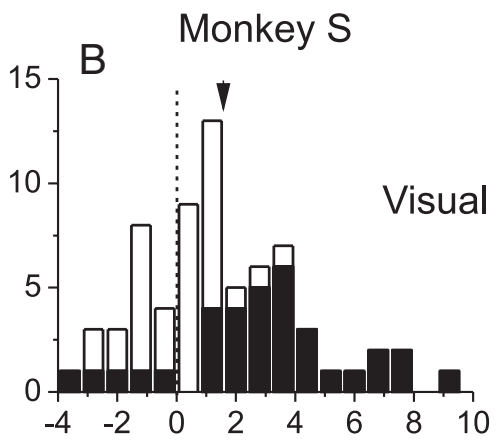

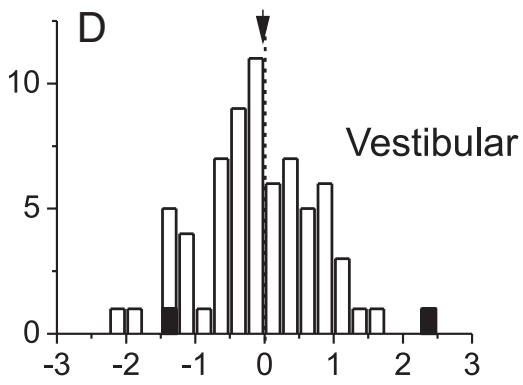

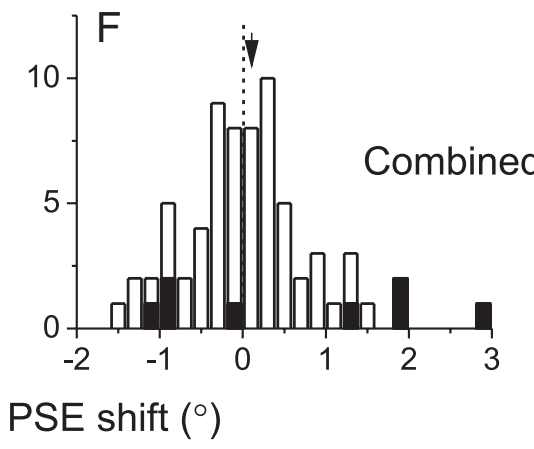

Induced PSE shift $\left(^{\circ}\right)$

Figure 4. Summary of microstimulation-induced shifts in the PSE of animals C and S. Data are shown separately for visual $(\boldsymbol{A}, \boldsymbol{B})$, vestibular $(\boldsymbol{C}, \boldsymbol{D})$, and combined $(\boldsymbol{E}, \boldsymbol{F})$ conditions. Positive PSE values represent shifts in the direction predicted by the heading tuning of MU activity. Negative PSE values represent shifts in the opposite direction. The filled and open bars represented significant and nonsignificant PSE shifts, respectively. Arrows, Mean PSE shift. The dashed vertical lines mark zero PSE shift.

strength of heading selectivity of MU activity (around straight ahead) was quantified using $d^{\prime}$, computed as follows:

$$
d^{\prime}=\frac{\left(R_{\text {left }}-R_{\text {right }}\right)}{\sqrt{\frac{\sigma_{\text {left }}^{2}+\sigma_{\text {right }}^{2}}{2}}}
$$

where $R_{\text {left }}, R_{\text {right }}, \sigma_{\text {left }}$ and $\sigma_{\text {right }}$ represent the mean responses and SDs in response to stimuli that were $22.5^{\circ}$ to the left and right of straight forward, respectively. These were the two headings closest to straight forward, thus providing our best estimate of how well neural activity discriminates between headings that vary around the forward reference used in the discrimination task.

Second, we quantified the monkey's behavioral performance by generating psychometric functions. Specifically, we plotted the proportion of "rightward" decisions as a function of heading direction (negative headings are leftward; positive headings are rightward). We then fit the data with a cumulative Gaussian function (Gu et al., 2007, 2008) that included two free parameters: (1) the psychophysical threshold was taken as the SD of the Gaussian fit and corresponded to $84 \%$ correct performance, and (2) the point of subjective equality (PSE) was taken as the mean of the Gaussian fit, corresponding to the heading angle that yielded $50 \%$ rightward and 50\% leftward choices. In microstimulation experiments, the significance of changes in PSE and threshold were assessed by a probit regression (Britten and van Wezel, 1998, 2002). 


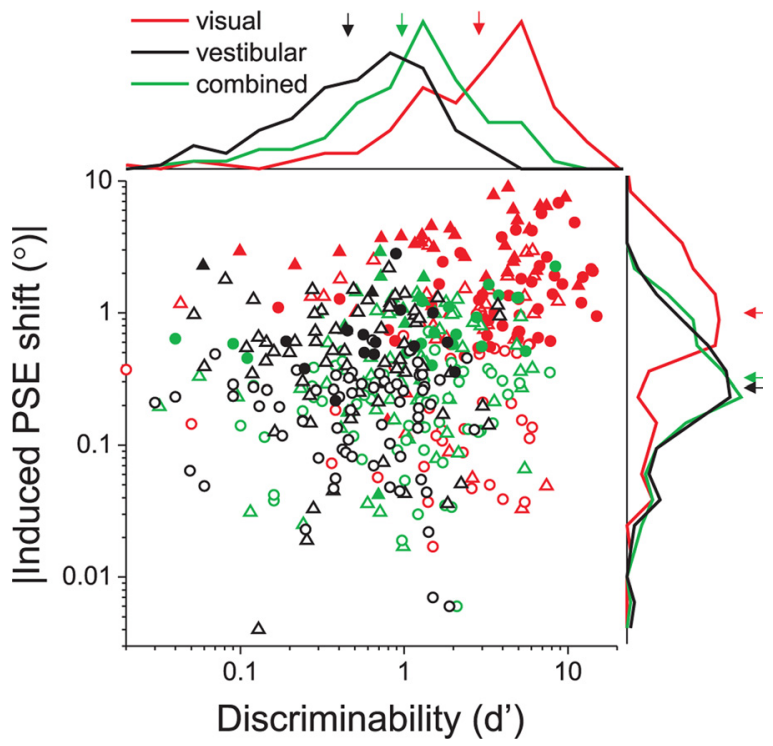

Figure 5. Relationship between the size of microstimulation effect (induced PSE shift) and MU discriminability for heading. For each stimulation site, a $d^{\prime}$ value that characterizes heading selectivity around straight forward was computed from the responses at -22.5 and $+22.5^{\circ}$. The filled and unfilled symbols denote significant and nonsignificant PSE shifts, respectively (monkey C, circles; monkey S, triangles). Data are shown separately for visual (red), vestibular (black), and combined (green) conditions. The top and right panels show marginal distributions of $\left|d^{\prime}\right|$ and $|\triangle P S E|$ (on log scales). The arrows mark the respective geometric means.

Predicted psychophysical thresholds for the combined condition, assuming optimal (maximum-likelihood) cue integration, were computed as follows (Ernst and Banks, 2002; Gu et al., 2008):

$$
\sigma_{\text {prediction }}=\sqrt{\frac{\sigma_{\text {vestibular }}^{2} \times \sigma_{\text {visual }}^{2}}{\sigma_{\text {vestibular }}^{2}+\sigma_{\text {visual }}^{2}}},
$$

where $\sigma_{\text {vestibular }}$ and $\sigma_{\text {visual }}$ represent psychophysical thresholds for the vestibular and visual conditions, respectively. According to Equation 2, the threshold in the combined condition, $\sigma_{\text {prediction, }}$ is lower than the lowest single-cue threshold, and the maximum predicted improvement (a factor of $\sqrt{ } 2$ ) occurs when the two single cues have equal reliability $\left(\sigma_{\text {vestibular }}=\sigma_{\text {visual }}\right)$. Thus, in preliminary testing for each animal, we found the motion coherence for which visual and vestibular thresholds were approximately matched (for details, see Gu et al., 2008). All electrical microstimulation experiments and most chemical inactivation experiments used the matched visual motion coherence for each animal: $38 \%$ (animal C), 26\% (animal S), and 70\% (animal J). However, because inactivation effects were substantially larger for visual than vestibular heading discrimination, visual and vestibular thresholds no longer matched after MSTd was inactivated. Thus, it was practically more difficult to examine whether animals were capable of combining visual and vestibular cues optimally (i.e., according to Eq. 2) following inactivation of MSTd. For this reason, an additional set of inactivation experiments was performed at $100 \%$ visual motion coherence to better match visual and vestibular sensitivity after MSTd inactivation, thus allowing us to test more precisely whether optimal cue integration (Eq. 2) persisted after inactivation.

Finally, we also tested whether inactivation of MSTd compromises the monkey's ability to reweight visual and vestibular signals from trial to trial according to relative cue reliability (Fetsch et al., 2009). In these experiments, a conflict angle $\left(\Delta= \pm 3^{\circ}\right)$ was introduced between the visual and vestibular heading directions in the combined condition $(+\Delta$ means vestibular heading was leftward relative to the visual heading, and $-\Delta$ means vestibular heading was rightward relative to the visual heading). Visual cue reliability was varied by manipulating visual motion coherence (20 and 70\%). All stimulus conditions were interleaved within a single block of trials (for more details, see Fetsch et al., 2009).
After fitting psychometric functions to the behavioral data from the cue-conflict task, two sets of weights were computed (Fetsch et al., 2009). The "predicted" weights, computed from the single-cue thresholds, describe how an optimal observer would weight the visual and vestibular cues as follows:

$$
\begin{aligned}
w_{\text {vest_pred }} & =\frac{1 / \sigma_{\text {vestibular }}^{2}}{1 / \sigma_{\text {vestibular }}^{2}+1 / \sigma_{\text {visual }}^{2}} \\
w_{\text {vest_pred }} & =\frac{1 / \sigma_{\text {visual }}^{2}}{1 / \sigma_{\text {vestibular }}^{2}+1 / \sigma_{\text {visual }}^{2}} .
\end{aligned}
$$

The "actual" weights were computed from the PSE measurements derived from psychometric functions in the cue-conflict conditions, after compensating for any overall bias in behavior by subtracting the PSE in the $\Delta=0^{\circ}$ case $\left(\mu_{0}\right)$, as follows:

$$
\begin{aligned}
& w_{\text {vest_actual }}=\frac{\mu-\mu_{0}+\frac{\Delta}{2}}{\Delta} \\
& w_{\text {vis_actual }}=1-w_{\text {vest_actual }} .
\end{aligned}
$$

The actual weights characterize how the animal weighted the visual and vestibular cues when they were placed in conflict. Actual weights were computed separately for $+\Delta$ and $-\Delta$ conflicts and then averaged for a given coherence level.

\section{Results}

Electrical microstimulation effects on heading discrimination The animals' behavior was monitored while low-amplitude electrical current (20 $\mu \mathrm{A}$ peak), which was tapered to follow the waveform of stimulus velocity (Fig. $1 B$ ), was delivered on onehalf of the trials through a microelectrode placed in area MSTd (see Materials and Methods). If visual and/or vestibular signals in MSTd are used by the monkey to judge heading, microstimulation is expected to add a net signal to the local circuit, thus resulting in a shift of the psychometric function toward the preferred heading of MU activity recorded at the stimulation site (Salzman et al., 1990, 1992; Murasugi et al., 1993; Celebrini and Newsome, 1995; Nichols and Newsome, 2002; Cohen and Newsome, 2004; Afraz et al., 2006; Uka and DeAngelis, 2006). Thus, microstimulation is mainly expected to affect the PSE, or bias, of the psychometric function, with little or no effect on the slope of the function (Fig. 1C).

During each experiment, we first mapped the heading tuning of MU activity at multiple locations, $\sim 100 \mu \mathrm{m}$ apart, along each electrode penetration. When we found a span of at least $200-300$ $\mu \mathrm{m}$ with consistent heading tuning for either the visual or vestibular condition (for details, see Materials and Methods), we chose a location with strong tuning near the middle of this span as the stimulation site. MU tuning for two example stimulation sites is illustrated in Figure 2. The MU tuning in Figure $2 \mathrm{~A}$ was significant for all stimulus conditions (vestibular, $p=0.0037$; visual low coherence, $p \ll 0.001$; visual high coherence, $p \ll 0.001$; combined, $p \ll 0.001$, ANOVA). In Figure $2 B$, the MU tuning was significant only for the visual and combined conditions (vestibular, $p=0.5$; visual low coherence, $p \ll 0.001$; visual high coherence, $p \ll 0.001$; combined, $p \ll 0.001$, ANOVA). Across all stimulation sites tested, MU activity was significantly tuned for 145 of 150 cases in the visual condition (at high coherence), 59 of 150 cases in the vestibular condition, and 100 of 150 cases in the combined condition. When visual heading tuning was compared between sites within the same electrode penetration, correlation coefficients were high for nearby sites, but low for sites separated by $>300 \mu \mathrm{m}$, as illustrated in Figure $3 A$ (median Pearson's correlation coefficients: $r=0.82$ for $100 \mu \mathrm{m} ; r=0.64$ for $200 \mu \mathrm{m}$; 
$r=0.11$ for $300 \mu \mathrm{m} ; r=0.14$ for $400 \mu \mathrm{m})$. The median correlation coefficient was significantly greater than zero for separations of $100 \mu \mathrm{m}(p \ll 0.001$, sign test $)$ and $200 \mu \mathrm{m}(p \ll 0.001$, sign test $)$, but not for $300 \mu \mathrm{m}(p=0.7)$ or $400 \mu \mathrm{m}(p=$ 0.3 ). This is consistent with previous reports showing clustering of optic flow tuning in MSTd (Tanaka et al., 1986; Geesaman et al., 1997; Britten, 1998; Chen et al., 2008). The vestibular tuning of MU activity showed a similar but weaker trend (Fig. $3 B$ ), with median correlation coefficients of $r=0.29$ at $100 \mu \mathrm{m}(p \ll 0.001)$, $r=0.28$ at $200 \mu \mathrm{m}(p \ll 0.001), r=0.19$ at $300 \mu \mathrm{m}(p=0.0044)$, and $r=0.08$ at $400 \mu \mathrm{m}(p=0.8)$.

For the example stimulation site illustrated in Figure $2 A$, the preferred heading of MU was leftward (negative). If the monkey uses MSTd activity to judge heading, electrical microstimulation of this site should increase the evidence in favor of leftward headings, as seen by the decision process. As a result, the monkey should be biased toward making more leftward choices, which would result in a rightward shift of the psychometric function (Fig. 1C). Indeed, during microstimulation trials in the visual condition, the monkey made leftward decisions much more frequently than during nonstimulated trials, resulting in a large $\left(6.79^{\circ}\right)$ rightward shift of the psychometric function (Fig. $2 C, p \ll 0.001$, probit regression; compare solid and dashed red lines). In contrast to this large shift in the visual condition, the vestibular psychometric curve shifted to the right only by $0.21^{\circ}$, which was not significant $(p>0.6$; Fig. 2C, black). Finally, the rightward shift of the psychometric function in the combined condition was equivalent to $1.41^{\circ}$ of heading ( $p \ll 0.001$; Fig. $2 C$, green). Importantly, the direction of the psychometric curve shift for all stimulus conditions was consistent with the heading preference of MU activity at the stimulation site.

In the experiment of Figure $2 B$, MU activity had a rightward (positive) heading preference. As a result, microstimulation of this site is expected to bias the monkey toward making more rightward choices. Consistent with this prediction, the monkey made more rightward choices during microstimulation, resulting in a leftward shift of the psychometric function. This shift was robust and highly significant in the visual condition $\left(3.76^{\circ}, p \ll 0.001\right.$, probit regression; Fig. $2 D$, red), but was not significant for the vestibular $\left(0.29^{\circ}, p>0.7\right.$; Fig. $2 D$, black $)$ and combined $\left(0.35^{\circ}, p=0.13\right.$; Fig. $2 D$, green $)$ conditions.

Figure 4 provides a population summary of shifts of the psychometric functions for all microstimulation experiments performed in two animals (monkey C, $n=81$; monkey $S, n=69$ ). The sign of the stimulation-induced shift was assigned to be positive if its direction was as predicted from the heading preference of MU activity at the particular stimulation site; a negative sign indicates that the psychometric curve shifted in the direction opposite to that predicted from MU tuning. In the visual stimulus condition (Fig. $4 A, B$ ), microstimulation of MSTd produced a shift in the predicted direction for most (110 of 150; 73.3\%) sites, and the shift was statistically significant for 70 of $110(63.6 \%)$ of these experiments. Mean shifts of the psychometric curve were significantly greater than 0 for both animals ( $p \ll 0.001, t$ test), averaging $0.89^{\circ}$ for monkey $\mathrm{C}$ and $1.52^{\circ}$ for monkey S. In contrast, the average induced shifts in the vestibular condition $(0.08$ and $-0.06^{\circ}$; Fig. $4 C, D$, respectively) were not significantly different from 0 ( $p>0.5, t$ tests). Moreover, microstimulation elicited a significant shift for only 16 of $150(10.7 \%)$ sites in the vestibular condition. Among the significant effects, only one-half (7 of 16) were consistent with the heading preference of MU activity at the corresponding stimulation sites.

Finally, for the combined stimulus condition (Fig. 4E,F), microstimulation generated effects that were intermediate between those of the two single-cue conditions. For monkey C, the mean shift of the psychometric function was significant in the predicted direction (mean $=0.27 ; p \ll 0.001, t$ test). For monkey $\mathrm{S}$, the average shift was also in the predicted direction but was not significantly different from 0 ( mean $=0.085 ; p=0.38, t$ test $)$. Over- 

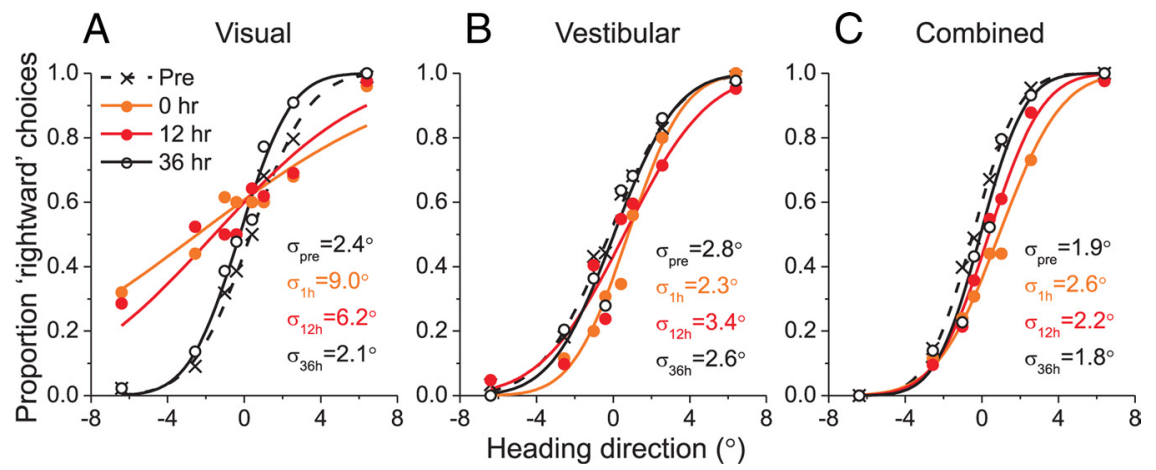

Figure 7. An example inactivation experiment. Psychometric functions were collected during four separate sessions: a control block before the day of muscimol injection (Pre, dashed curves and cross symbols), a block immediately after inactivation ( $0 \mathrm{~h}$, orange), a block $12 \mathrm{~h}$ after inactivation ( $12 \mathrm{~h}$, red), and a recovery block $36 \mathrm{~h}$ after inactivation ( $36 \mathrm{~h}$, black solid curves and open symbols). Psychometric functions are shown for each of visual $(\boldsymbol{A})$, vestibular $(\boldsymbol{B})$, and combined $(\boldsymbol{C})$ conditions. These data were collected following four bilateral injections of muscimol ( 2 in each hemisphere), each having a volume of $2 \mu$ l and a concentration of $10 \mu \mathrm{g} / \mu \mathrm{l}$ (see Materials and Methods).

all, significant shifts were observed in 31 of 150 (21\%) cases, and the majority were in the predicted direction $(n=24 ; 77 \%)$. These results illustrate that, whereas microstimulation had robust effects on heading judgments based on optic flow, effects were substantially weaker in the vestibular and combined conditions.

Can the weak microstimulation effects observed in the vestibular and combined conditions be explained by weak heading tuning in MU responses? Weak MU tuning likely reflects weak selectivity of single neurons and/or weak clustering of neurons according to heading preference. When MU tuning is weak, microstimulation is unlikely to inject a clean heading signal into the neural representation, and therefore may not bias choices. To quantify how well MU activity is tuned to heading around straight forward, we computed $d^{\prime}$ (see Materials and Methods) from the two stimulus directions closest to straight ahead $\left( \pm 22.5^{\circ}\right)$. A $d^{\prime}$ value close to 0 indicates that MU activity is roughly equal for $\pm 22.5^{\circ}$ headings, thus indicating weak tuning around straight ahead. In contrast, a large absolute $d^{\prime}$ value indicates that the difference in MU activity between leftward and rightward headings is large relative to the SD of the responses. We found significant differences in $d^{\prime}$ across stimulus conditions $\left(p \ll 0.001\right.$, one-way ANOVA). The geometric mean value of $d^{\prime}$ was greatest in the visual condition (2.46), smallest in the vestibular condition (0.49), and intermediate in the combined condition (0.96), as illustrated by the marginal distributions across the top of Figure 5. To examine whether variations in $d^{\prime}$ across stimulus conditions could account for differences in the efficacy of microstimulation across stimulus conditions, we used analysis of covariance (ANCOVA) to examine the dependence of microstimulation effects on $d^{\prime}$ within and across stimulus conditions, as illustrated in Figure 5. Across all stimulus conditions, there was a significant correlation such that microstimulation of recording sites with greater $d^{\prime}$ produced larger PSE shifts in the monkey's behavior $(r=0.41, p=0.0091$, covariate effect, ANCOVA). Indeed, inspection of Figure 5 reveals that most of the significant effects of microstimulation in the visual condition occurred at recording sites having $d^{\prime}$ values greater than nearly all $d^{\prime}$ values seen in the vestibular condition.

Importantly, the ANCOVA analysis revealed no significant interaction between $d^{\prime}$ and stimulus condition $(p=0.15$, ANCOVA), indicating that there was no significant evidence for different relationships between PSE shift and $d^{\prime}$ across the three stimulus conditions. This suggests that weak effects of micro- stimulation in the vestibular and combined conditions may be mainly attributed to lack of robust MU heading tuning in these conditions. Note, however, that there was also a significant main effect of stimulus condition $(p \ll 0.001$, ANCOVA) when the interaction term was included in the model. This suggests either that the relationship between PSE shift and $d^{\prime}$ is nonlinear or that other factors in addition to $d^{\prime}$ also contribute to the heterogeneous microstimulation effects seen across stimulus conditions.

Whereas microstimulation produced systematic effects on PSEs, it had little effect on psychophysical thresholds. Only $7.3 \%$ (11 of 150 ), $13.3 \%$ (20 of 150), and $6 \%$ (9 of 150) of experiments revealed significant effects of microstimulation on thresholds for the visual, vestibular, and combined conditions, respectively $(p<$ 0.05 , probit regression). At the population level, there was no difference in average thresholds between stimulated and nonstimulated trials for monkey $\mathrm{C}$ in all stimulus conditions $(p>$ 0.16 , paired $t$ test; Fig. $6 A$ ). This also held true in the vestibular $(p=0.15)$ and combined $(p=0.7)$ conditions for monkey $\mathrm{S}$, whereas visual thresholds increased significantly by $\sim 20 \%$ during microstimulation $(p \ll 0.001$; Fig. $6 B$ ). This increase in threshold for monkey $S$ could be due to the large PSE shifts observed during some microstimulation experiments in this animal. Indeed, there was a significant correlation between the change in threshold and PSE shift (Fig. $6 C ; r=0.35, p \ll 0.001$, Spearman's rank correlation). That is, in sessions when a large PSE shift occurred, there was also a larger than average change in threshold. This is not surprising: a very large PSE shift would result in a flattened psychometric function over the heading range tested, leading to a high threshold. In addition, psychometric functions having very large PSE shifts are not well sampled around the steep portion of the curve (Fig. $2 \mathrm{C}$, red), which may also cause overestimation of the threshold.

Consistent with our previous findings (Gu et al., 2008; Fetsch et al., 2009), Figure 6 shows that both animals integrated visual and vestibular cues in a statistically optimal or near-optimal manner to improve heading discrimination performance. Average thresholds in the combined condition were significantly smaller than both single-cue thresholds ( $p \ll 0.001$, paired $t$ test) and similar to predictions from optimal cue integration (Fig. 6, "prediction"). Indeed, for monkey $\mathrm{C}$, combined and predicted thresholds were not significantly different ( $p=0.5$, paired $t$ test), whereas monkey $S$ showed modestly but significantly higher combined thresholds than predicted from optimal cue integration $(p<0.001$, paired test). Notably, microstimulation of MSTd did not appear to change the optimality of cue combination seen in heading discrimination behavior. Combined and predicted thresholds were well correlated $(r=0.82, p \ll 0.001$, covariate effect, ANCOVA), and this correlation was not significantly different between stimulated and nonstimulated trials (Fig. $6 D ; p=$ 0.43 , interaction effect; $p=0.14$, group effect, ANCOVA). This result also held when data from each monkey were $z$-scored and combined before performing ANCOVA $(r=0.33, p \ll 0.001$, covariate effect; $p=0.7$, interaction effect; $p=0.1$, group effect, ANCOVA).

Previous studies have shown that MSTd neurons can have visual and vestibular tuning that is either congruent or opposite 

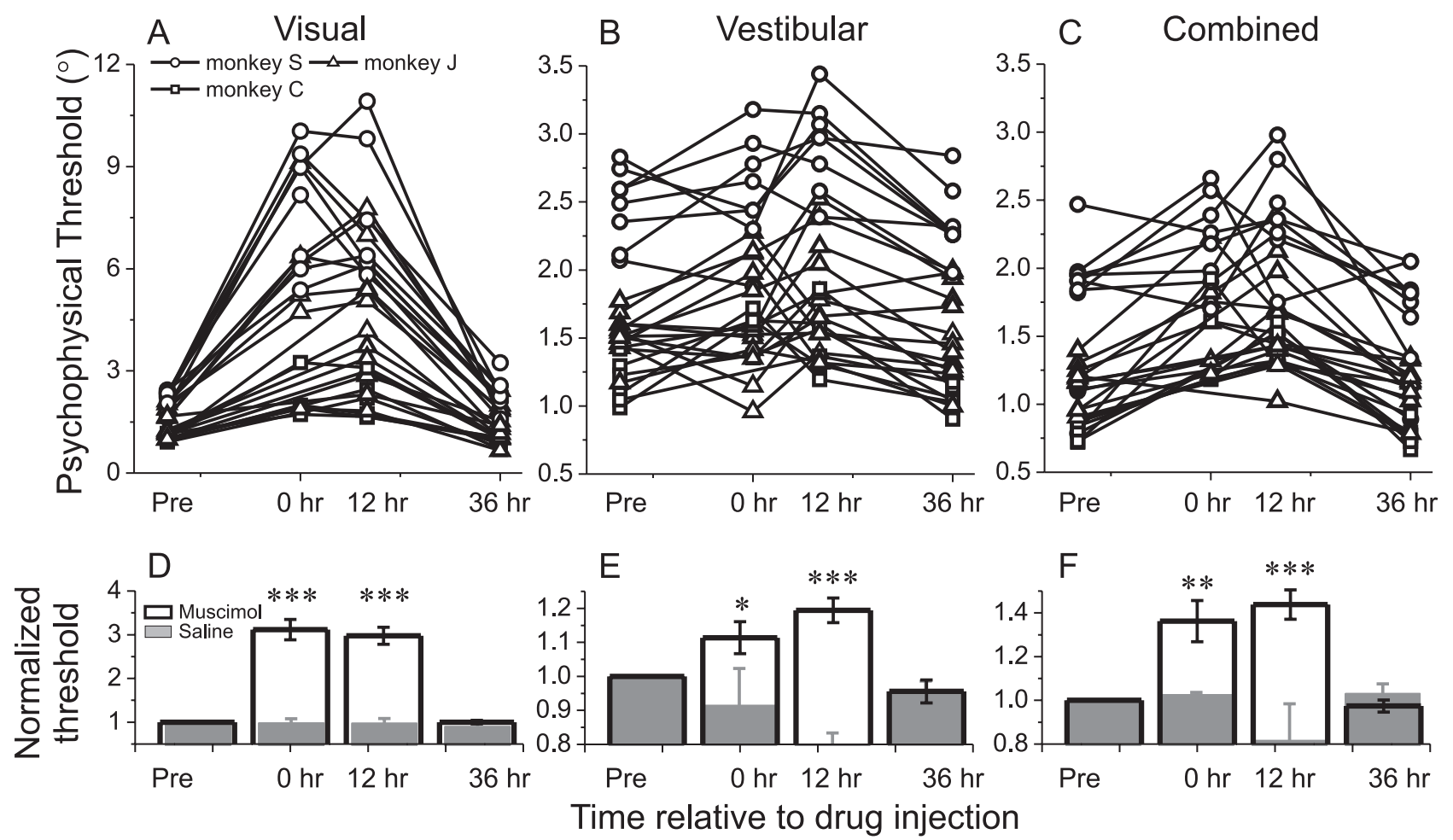

Figure 8. Summary of inactivation-induced increases in psychophysical thresholds of animals S (circles), J (triangles), and C (squares); data were collected following four bilateral muscimol injections (see Materials and Methods). Data are shown separately for visual $(\boldsymbol{A}, \boldsymbol{D})$, vestibular $(\boldsymbol{B}, \boldsymbol{E})$, and combined $(\boldsymbol{C}, \boldsymbol{F})$ conditions. The abscissa in each panel marks the four different time points at which data were collected: $\operatorname{Pre}, 0,12$, or $36 \mathrm{~h}$. Top panels $(\boldsymbol{A}-\boldsymbol{C}$ ) show data from individual experiments, whereas bottom panels ( $\boldsymbol{D}-\boldsymbol{F}$, open bars) show average normalized thresholds (normalized by dividing the threshold at each time point by that from the Pre block). The asterisks mark normalized thresholds significantly greater than unity $\left({ }^{*} p<0.05\right.$; $\left.{ }^{* *} p<0.01 ;{ }^{* * *} p<0.001\right)$. Error bars indicate SEM. For comparison, mean normalized thresholds from saline control injections are superimposed ( $\boldsymbol{D}-\boldsymbol{F}$, gray bars).

(Page and Duffy, 2003; Gu et al., 2006). Thus, it is important to investigate whether microstimulation effects depend on the congruency of visual/vestibular tuning. We characterized the tuning of MU responses at each stimulation site with a "congruency index" by computing the Pearson correlation coefficient between visual and vestibular tuning curves, such as those shown in Figure $2, A$ and $B$. The congruency index was broadly distributed within the range from -1 to +1 , with roughly equal numbers of sites having significantly congruent $(n=11 ; 7.3 \%)$ and opposite MU tuning ( $n=12 ; 8 \% ; p<0.05$, Pearson's correlation coefficient). Note that the numbers of sites with significantly congruent or opposite tuning was small due to the low incidence of significant vestibular tuning in MU responses. The average congruency index was $-0.09 \pm 0.03$ (mean \pm SEM), which was significantly different from zero ( $p=0.006, t$ test). Importantly, microstimulation-induced PSE shifts did not depend significantly on the congruency index, and this held true for all stimulus conditions (vestibular: $r=0.08, p=0.3$; visual: $r=0.06, p=0.5 ; r=0.07, p=0.4$, Spearman's rank correlation). Similarly, microstimulation effects on psychophysical threshold did not depend significantly on congruency (vestibular: $r=$ $-0.07, p=0.4$; visual: $r=-0.04, p=0.7 ; r=-0.12, p=0.14$, Spearman's rank correlation). Thus, there was no evidence that microstimulation effects depended on the congruency of visual and vestibular tuning, although the number of sites available to test for this dependency was limited.

In summary, microstimulation of area MSTd consistently and predictably biased heading percepts based on optic flow, but had no significant overall effect on heading judgments based on vestibular cues. Although this may appear to suggest that MSTd does not contribute to vestibular heading judgments, the vestibular data are inconclusive because the vestibular heading tuning of MU activity was generally weak. Thus, these results do not clearly distinguish between the absence of a causal contribution of MSTd to vestibular heading perception and an inability to sufficiently activate clusters of MSTd neurons with consistent vestibular heading preferences. To distinguish between these two possibilities, we turned to reversible chemical inactivation experiments.

\section{Chemical inactivation effects on heading discrimination}

Muscimol, a $\mathrm{GABA}_{\mathrm{A}}$ receptor agonist, was used to temporarily and reversibly inactivate large portions of area MSTd, either unilaterally or bilaterally. Unlike electrical microstimulation, this technique does not rely on local clustering of heading tuning because muscimol injections inactivate a region much larger than local clusters of heading-tuned neurons (Britten, 1998; Chen et al., 2008). For these reasons, inactivation of MSTd, unlike microstimulation, is expected to increase heading thresholds (flatten the psychometric function) rather than inducing a choice bias (Fig. 1D). The majority of the data were collected following four sequential injections of muscimol $(\sim 2 \mu \mathrm{l}$ volume per injection; $10 \mu \mathrm{g} / \mu \mathrm{l}$ concentration), two injections in each hemisphere, $\sim 2-3 \mathrm{~mm}$ apart (for details, see Materials and Methods). For each inactivation experiment, behavioral performance was measured in the visual, vestibular, and combined stimulus conditions (randomly interleaved) every day during a period of a week. On the first $2 \mathrm{~d}$ of each week (Monday and Tuesday), control data were collected before inactivation ("Pre"). On the day of the muscimol injections (typically a Wednesday afternoon), a short block of behavioral data was obtained immediately after inactivation (" $0 \mathrm{~h}$ "). On the morning of the following day (Thursday), a long block of heading discrimination was performed ("12 h"), fol- 

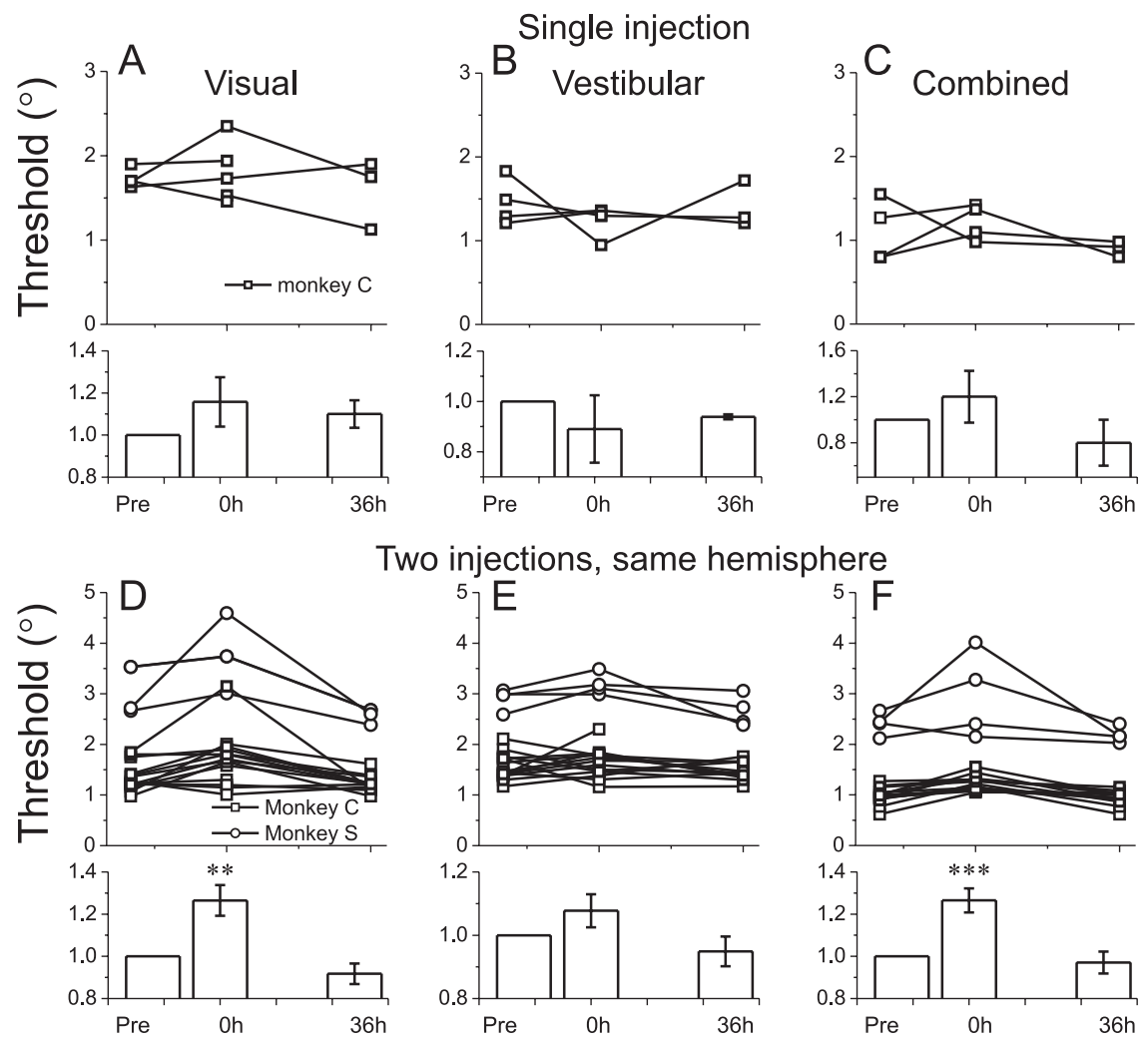

Two injections, same hemisphere
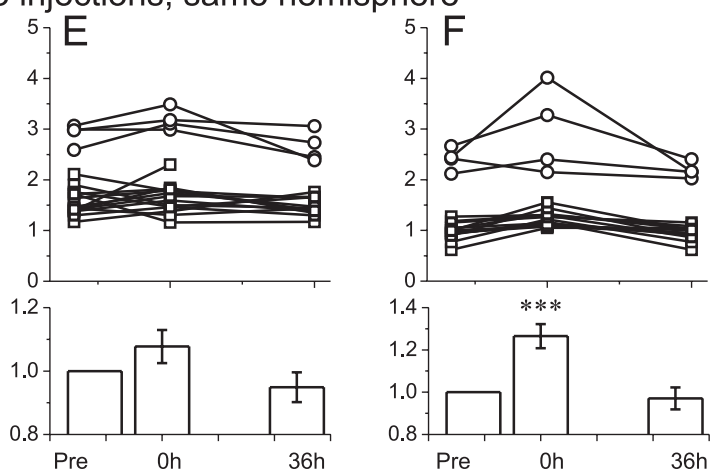

Two injections, opposite hemispheres
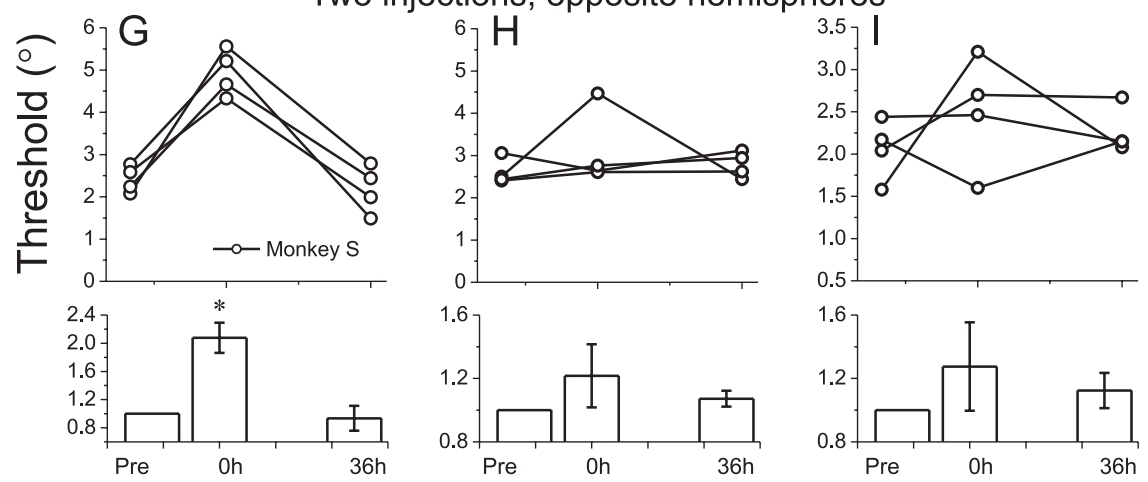

Time relative to drug injection

Figure 9. Summary of changes in psychophysical thresholds following various configurations of muscimol injections: $\boldsymbol{A}-\boldsymbol{C}$, single site; $\boldsymbol{D}-\boldsymbol{F}$, two injections in the same hemisphere; $\boldsymbol{G}-\boldsymbol{I}$, two injections, one per hemisphere. Each injection contained $2 \mu \boldsymbol{l}$ of muscimol at a concentration of $10 \mu \mathrm{g} / \mu \mathrm{l}$. Note that these alternative injection configurations were performed only in animals $\mathrm{C}$ and $\mathrm{S}$ before the four-site bilateral injections shown in Figure 7 , and $12 \mathrm{~h}$ data were not collected in these experiments. The format is as in Figure 8.

lowed by another long block of trials $36 \mathrm{~h}$ after inactivation (“36 h"), on Friday.

Results from a typical experiment (four injections bilaterally) are illustrated in Figure 7. The largest effects were seen in the visual condition, where inactivation of MSTd substantially deteriorated the monkey's behavioral performance (Fig. $7 A$ ), such that the heading threshold increased from $2.4^{\circ}$ preinjection (dashed black curve) to $9^{\circ}$ immediately following the injections (orange). The threshold remained high $\left(6.2^{\circ}\right) 12 \mathrm{~h}$ after inactivation (red) and recovered to preinjection levels $\left(2^{\circ}\right)$ at the $36 \mathrm{~h}$ time point (solid black curve). Inactivation also mildly impaired the monkey's performance in the vestibular and combined conditions, but these effects were weak compared with the visual condition (Fig. $7 B, C$ ).
Results from 27 inactivation experiments performed on three animals (monkey C, $n=7$; monkey S, $n=8$; monkey J, $n=12$ ) are summarized in Figure 8. Significant increases in psychophysical threshold were seen in all stimulus conditions, with the strongest effects on visual heading perception (Fig. $8 A-C$ ). To summarize the inactivation effects, Figure $8 D-F$ shows normalized thresholds obtained by dividing the threshold measured at each time point by the corresponding preinjection (Pre) threshold, and then pooling data across experiments and animals for each stimulus condition. Visual heading thresholds increased approximately threefold after inactivation ( 0 and $12 \mathrm{~h}$ time points; $p \ll 0.001, t$ test; Fig. $8 D)$, whereas increases in vestibular thresholds averaged $11.4 \%$ at $0 \mathrm{~h}(p=$ 0.02 ) and $19.4 \%$ at $12 \mathrm{~h}$ after injection $(p \ll 0.001)$ (Fig. 8E). Combined thresholds increased on average by $36.2 \%$ ( $p<$ $0.01)$ and $43.8 \%(p \ll 0.001)$ at the 0 and $12 \mathrm{~h}$ time points, respectively (Fig. $8 F$ ). For all stimulus conditions, mean heading thresholds measured $36 \mathrm{~h}$ after inactivation (36 h time point) were not significantly different from preinjection levels $(p>0.2, t$ test), indicating that the effects of muscimol on heading discrimination recovered completely within $2 \mathrm{~d}$ following the injection. In contrast, saline injections into area MSTd, performed using an identical protocol, did not significantly elevate heading thresholds in any stimulus condition ( $p>0.1, t$ test), as illustrated with gray bars in Figure $8 D-F$. The only significant departure from baseline performance following saline injections was a small decrease of vestibular thresholds at the $12 \mathrm{~h}$ time point, which was weakly significant $(p=0.03)$.

In summary, suppressing neural activity in area MSTd significantly and reversibly impaired heading discrimination. Effects were strongest for the visual condition, weakest for the vestibular condition, and intermediate for the combined condition. As all of the above findings were obtained following four bilateral injections of muscimol (two in each hemisphere), we further explored the dependency of the results on the extent of the inactivation. No significant effects were observed in any stimulus condition following a single $(\sim 2 \mu \mathrm{l})$ injection of muscimol into one hemisphere (Fig. 9A-C; $n=4$ ). Following two injections of muscimol into the same hemisphere, we observed modest but significant elevations of heading thresholds only for the visual and combined conditions (Fig. 9D-F; $n=14$ ) and these effects were substantially weaker than those seen in Figure 8. Finally, following bilateral muscimol injections (one per hemisphere), we observed robust effects on visual thresholds but no significant effects on vestibular or combined thresholds (Fig. 9G-I; $n=4$ ). Together with Figure 8, these results indicate that it is necessary to 

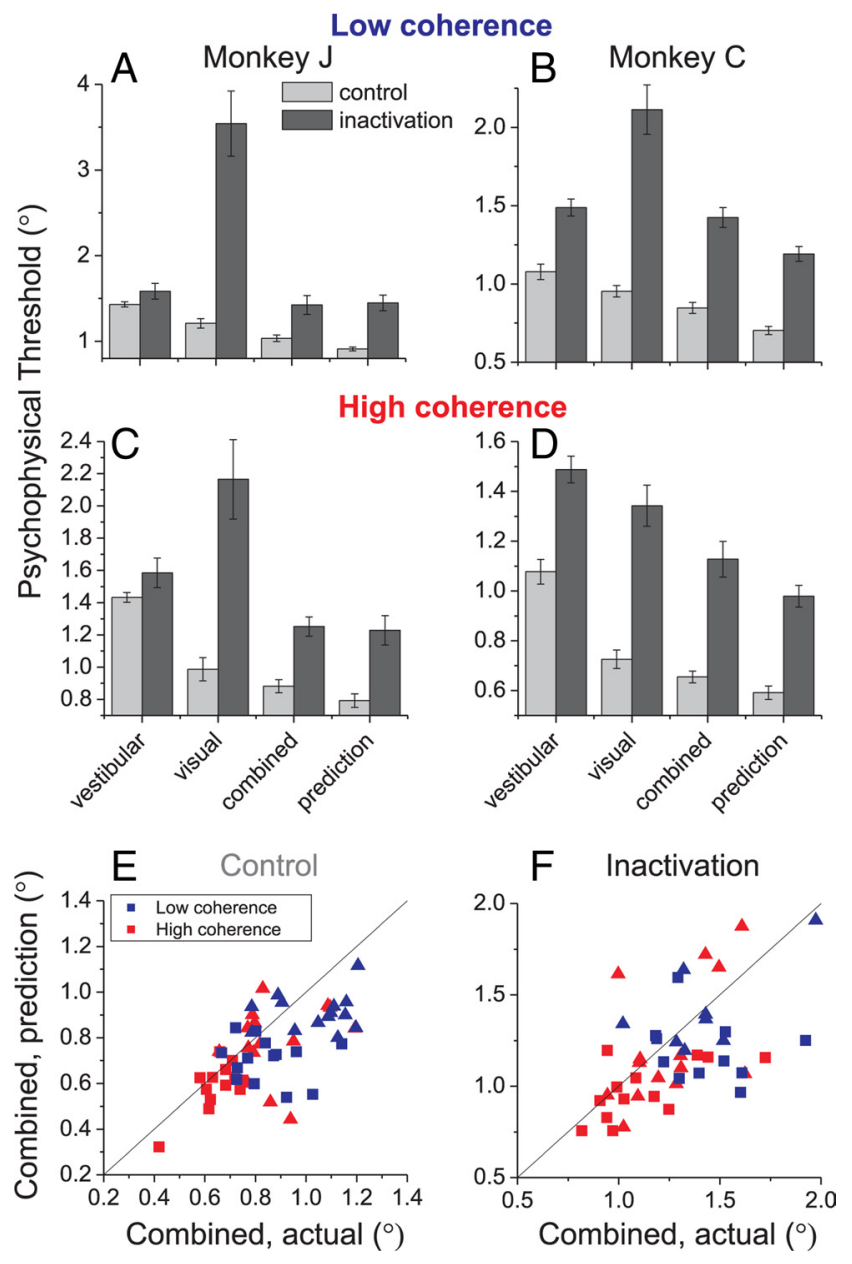

Figure 10. Cue integration effects before and after inactivation (4 injections, bilateral). Data for low coherence $(\boldsymbol{A}, \boldsymbol{B})$ and high coherence $(\boldsymbol{C}, \boldsymbol{D})$ from monkeys $J$ and $C$. Data are shown as mean ( $\pm S E M)$ threshold for the three stimulus conditions, along with the prediction from optimal cue integration (Eq. 2). The gray bars represent control thresholds (from Pre and $36 \mathrm{~h}$ sessions pooled together). The black bars represent inactivation thresholds (from 0 and $12 \mathrm{~h}$ blocks pooled together). $\boldsymbol{E}, \boldsymbol{F}$, Comparison between actual and predicted combined thresholds for the control and inactivation sessions, respectively. Blue symbols, Low coherence; red symbols, high coherence. Triangles, Monkey J; squares, monkey C.

inactivate relatively large portions of area MSTd bilaterally to observe robust effects on behavior (see Discussion).

Next, we examine whether MSTd inactivation impairs the monkey's ability to combine visual and vestibular cues nearoptimally to improve heading discrimination performance. Note that the disproportionally large deficit in visual thresholds (threefold elevation), compared with vestibular thresholds ( $\sim 20 \%$ elevation), alters the relative reliabilities of visual and vestibular cues following inactivation. Recall that matched single-cue thresholds maximize the opportunity to observe behavioral evidence of cue integration (Eq. 2) (Ernst and Banks, 2002; Gu et al., 2008). Without such matching of single-cue thresholds, predicted thresholds for the combined condition would be close to, and practically indistinguishable from, the best single-cue threshold, even if the cues are integrated optimally. Indeed, as shown in Figure $10 \mathrm{~A}$, the average combined threshold $\left(1.41 \pm 0.1^{\circ}\right)$ for animal $\mathrm{J}$ after inactivation (black bars) is statistically indistinguishable from both the best single-cue threshold (1.58 $\pm 0.08^{\circ}$; $p=0.11$, paired $t$ test $)$ and the optimal prediction $\left(1.42 \pm 0.08^{\circ}\right.$; $p=0.9$, paired $t$ test). Similarly, for animal C (Fig. $10 \mathrm{~B}$ ), the combined threshold $\left(1.43 \pm 0.07^{\circ}\right)$ is not significantly different from the best single-cue threshold $\left(1.49 \pm 0.06^{\circ} ; p=0.6\right.$, paired $t$ test) and is only marginally greater than the optimal prediction $\left(1.2 \pm 0.06^{\circ} ; p=0.03\right.$, paired $t$ test $)$.

Thus, to distinguish whether animals can still integrate heading cues near-optimally after inactivation of area MSTd or whether they simply follow the more reliable (vestibular) cue, another set of inactivation experiments was performed with visual motion coherence increased to 100\%. As shown in Figure 10, $C$ and $D$, visual and vestibular thresholds were better matched after inactivation. At $100 \%$ coherence, the average combined threshold for animal $\mathrm{J}\left(1.25 \pm 0.06^{\circ}\right)$ was now significantly smaller than the best single-cue threshold $\left(1.5 \pm 0.1^{\circ} ; p=0.016\right.$, paired $t$ test) and was not distinguishable from the optimal prediction $\left(1.23 \pm 0.09^{\circ} ; p=0.7\right.$, paired $t$ test; Fig. $\left.10 C\right)$. For animal $\mathrm{C}$, the pattern of thresholds following inactivation at high coherence was now similar to that seen under normal conditions at low coherence: the combined threshold $\left(1.13 \pm 0.07^{\circ}\right)$ was significantly smaller than the best single-cue threshold $\left(1.34 \pm 0.08^{\circ} ; p=0.02\right.$, paired $t$ test $)$, but larger than the optimal prediction $\left(0.98 \pm 0.04^{\circ} ; p=0.02\right.$, paired $t$ test $)$. Thus, at high motion coherence, both monkeys demonstrated improved heading discrimination in the combined condition following inactivation, and the pattern of results was similar to the control data of Figure 6.

Scatterplots comparing predicted (ordinate) and actual (abscissa) combined thresholds for both low (blue) and high (red) coherence are shown in Figure 10, E and $F$, for control and inactivation sessions, respectively. Considering data from both coherences together, predicted and actual thresholds were significantly correlated ( $p \ll 0.001$, covariate effect, ANCOVA). Importantly, the slope of this relationship was not significantly different between inactivation and control conditions $(p=0.9$, interaction effect, ANCOVA). Thus, although single-cue thresholds increase following inactivation of MSTd, monkeys continue to be able to integrate visual and vestibular cues near-optimally to improve the precision of heading discrimination performance.

Finally, we examined whether monkeys can still reweight visual and vestibular inputs in proportion to their reliability (Fetsch et al., 2009), following inactivation of MSTd. We trained one animal (monkey S) to perform the heading discrimination task as described above, but with a small conflict angle $\left(\Delta=3^{\circ}\right)$ introduced between the visual and vestibular inputs during cue combination (Fig. 11A) (Fetsch et al., 2009). The relative reliability of the two cues was controlled by varying the visual motion coherence (20 and $70 \%)$ randomly across trials (Fig. $11 B-D)$. We then computed an actual vestibular weight $\left(w_{\text {vest_actual }}\right)$ by comparing how much the PSE in the combined condition was shifted by the introduction of cue conflict (for details, see Materials and Methods). In the control condition before injection, the actual vestibular weight $\left(w_{\text {vest actual }}\right)$ was significantly greater at low coherence (0.86) than at high coherence (0.49), and this difference was significant $(p=0.0087$, paired $t$ test; Fig. $11 E)$. Moreover, $w_{\text {vest_actual }}$ was significantly correlated with the predicted vestibular weight $\left(w_{\text {vest_pred }}\right)$ that was computed from single-cue thresholds (see Materials and Methods; $r=0.67, p=0.0087$, Spearman's rank correlation; Fig. $11 F$ ). These observations in the control condition are consistent with previous findings in both monkeys and humans (Fetsch et al., 2009).

Following inactivation of MSTd, due to the greater effect of muscimol on visual heading thresholds (Figs. 8-10), the predicted vestibular weight, $w_{\text {vest pred }}$, increased for both low motion coherence trials (Fig. $11 F, G$; control: mean $=0.59$; inactivation: mean $=0.75, p=0.003, t$ test; blue symbols) and high motion 
A
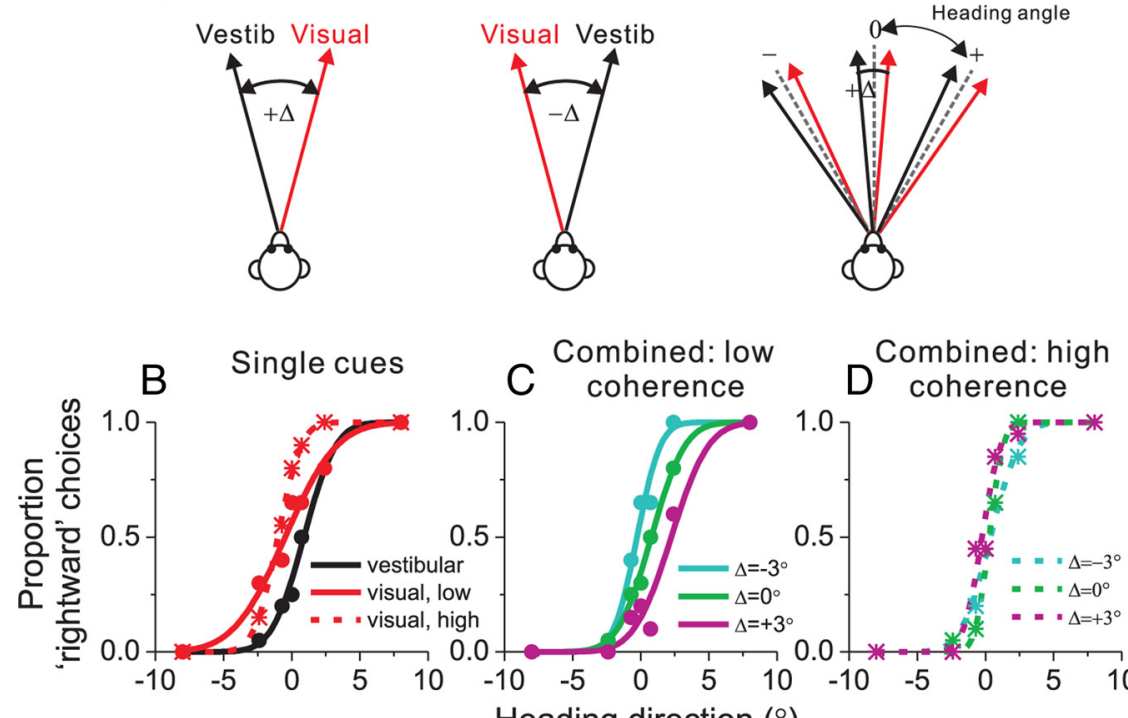

Combined: high
coherence

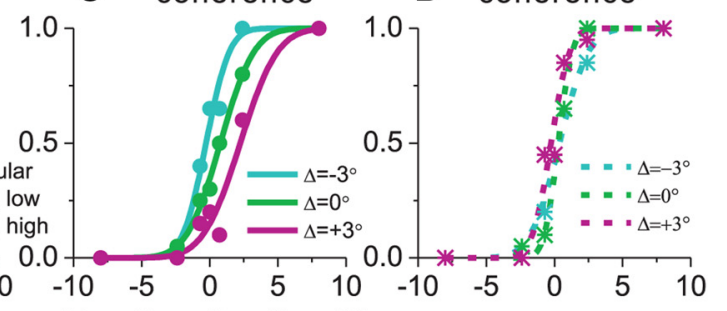

Heading direction $\left({ }^{\circ}\right)$
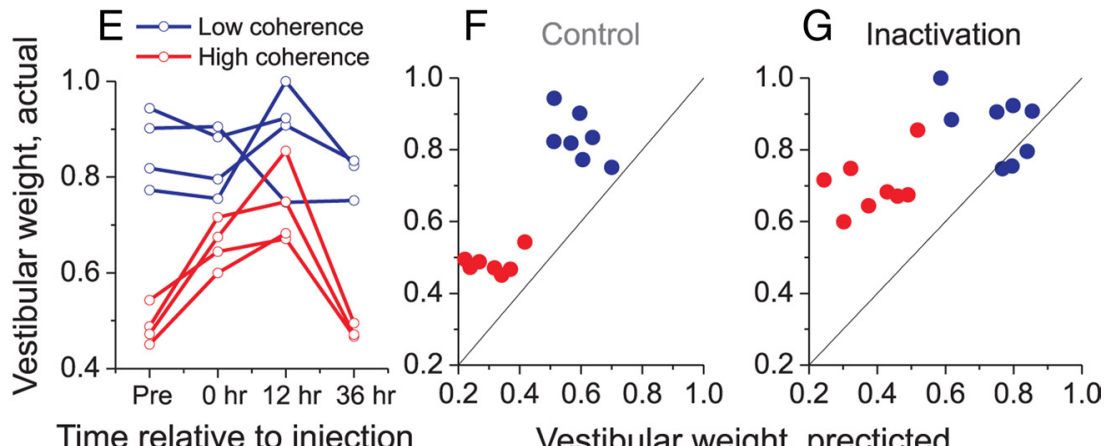

Time relative to injection

Vestibular weight, precticted

Figure 11. Cue reweighting effects before and after inactivation (4 injections, bilateral). $\boldsymbol{A}$, Stimulus arrangement during cue-conflict trials. Positive $\Delta$ (left) indicates that the visual heading is deviated to the right of the vestibular heading, and vice versa for negative $\Delta$ (middle). For a given $\Delta$ (right), heading angle was defined as the midpoint between the visual and vestibular headings, which were varied together in fine steps around straight forward. $\boldsymbol{B}-\boldsymbol{D}$, Example psychometric functions from one experimental session in the single-cue conditions $(\boldsymbol{B})$, combined condition at low motion coherence $(\boldsymbol{C})$, and combined condition at high motion coherence $(\boldsymbol{D})$. $\boldsymbol{E}$, The actual vestibular weight ( $\left.\boldsymbol{w}_{\text {vest_actual }}\right)$ (see Materials and Methods) as a function of time relative to drug injection for four inactivation experiments performed with monkey $S$. Each experiment contains interleaved low (20\%; blue) and high (70\%; red) motion coherences. F, G, Comparison between actual and predicted vestibular weights (see Materials and Methods) estimated from control and inactivation sessions, respectively. Blue symbols, Low coherence; red symbols, high coherence.

coherence trials (control: mean $=0.31$; inactivation: mean $=$ $0.39, p=0.089$; Fig. $11 F, G$, red symbols). Thus, we expected to see higher actual vestibular weights, $w_{\text {vest_actual }}$, if the animal still reweighted cues according to their reliability following inactivation. Consistent with this hypothesis (Fig. $11 E$ ), we observed a significant increase in $w_{\text {vest_actual }}$ after injection for the high motion coherence ( $0 \mathrm{~h}$ vs preinjection: $p=0.009$, paired $t$ test; $12 \mathrm{~h}$ vs preinjection: $p=0.017$ ), but not for the low coherence ( $0 \mathrm{~h}$ vs preinjection: $p=0.16 ; 12 \mathrm{~h}$ vs preinjection: $p=0.6$; Fig. $11 E$ ). The lack of effect of muscimol on $w_{\text {vest_actual }}$ at low coherence may be at least partly due to a ceiling effect, since the actual weights were already close to 1 before injection. Most importantly, there was still a robust correlation between actual and predicted vestibular weights following inactivation of MSTd $(r=0.69, p=0.003$, Spearman's rank correlation; Fig. $11 G)$, indicating that the animal still reweights cues in proportion to their reliability when MSTd is partially inactivated. Together with the data of Figure 10, this result indicates that near-optimal cue integration is largely preserved after inactivation of MSTd.
Heading angle

Simulation of lesion effects suggests contributions from other areas

Muscimol inactivation produced a peculiar pattern of results: threshold elevations in the combined condition were intermediate between those of the visual and vestibular conditions, yet the optimality of cue integration was largely conserved. The implications of this pattern of results are not immediately obvious; thus, we performed simple simulations to gain further insight.

We first examined how inactivation of a subset of a single multisensory area (like MSTd) would be expected to affect behavioral sensitivity across cue conditions. For this purpose, we simulated a population of 1000 MSTd-like neurons with congruent visual and vestibular heading tuning. Each unit had cosine tuning for heading, and heading preferences were uniformly distributed in azimuth. Visual heading tuning curves had larger amplitudes than vestibular tuning curves (Fig. 12A, left) to mimic the stronger visual heading tuning seen in MSTd (Gu et al., 2006, 2007, 2010), and all units had independent Poisson spiking statistics. In the combined condition, the response of each unit was the simple arithmetic sum of its visual and vestibular responses. Population sensitivity was quantified by computing Fisher information $(\mathrm{Gu}$ et al., 2010) and was transformed into predicted behavioral thresholds for each cue condition.

When decoding responses from all neurons in this model MSTd, predicted discrimination thresholds rise with the proportion of neurons that were "inactivated" (not contributing to Fisher information; Fig. $12 \mathrm{~B}$, left). Importantly, however, predicted thresholds rise proportionately across all stimulus conditions, such that the ratios of thresholds across pairs of stimulus conditions (Fig. 12C, left) remain constant. Although this scenario correctly predicts that optimal cue combination persists following inactivation, it is grossly inconsistent with the overall pattern of results we obtained by inactivating MSTd. In the real data (Fig. 8), inactivation effects were much larger for the visual than vestibular condition, such that the ratio of visual/vestibular thresholds was not constant following inactivation. Therefore, our findings do not appear to be consistent with scenarios in which a single multisensory area (MSTd) contributes to heading perception.

We therefore expanded the simulations and incorporated a second population of neurons that have vestibular-dominated heading tuning (but are otherwise identical; Fig. 12 A, right). This population mimics the stronger vestibular heading tuning we have observed in the visual posterior sylvian (VPS) area, for example (Chen et al., 2011b). In this simulation, the decoder reads out signals from neurons in both populations, but inactivation only removes a subset of units from the MSTd-like population. Results of this simulation are qualitatively very similar to the data of Figure 8 . Inactivation of the 
model MSTd area now produces much greater effects on visual heading discrimination than vestibular discrimination (Fig. $12 \mathrm{~B}$, right), with combined thresholds showing an intermediate effect. The ratio of visual/vestibular thresholds therefore increases following inactivation (Fig. 12C, right), consistent with the data we observed. Importantly, this model again exhibits optimal cue integration behavior despite the loss of some units.

This simple simulation suggests that our experimental observations are consistent with a family of scenarios in which other brain areas having vestibular-dominant tuning, such as VPS (Chen et al., 2011b), also contribute to heading perception in conjunction with area MSTd. The basic pattern of results in this simulation is quite robust to variations in the single-unit tuning curves, and the results hold qualitatively as long as one area (MSTd) is visual-dominant and the other area is vestibular-dominant. This is true even if the second area has essentially no visual heading tuning at all, like we have found in parietoinsular vestibular cortex (PIVC) (Chen et al., 2011a). Thus, our experimental findings appear to be consistent with the operation of a network of areas with a substantial range of cue dominance across areas.

\section{Discussion}

We have shown that manipulation of neuronal activity in area MSTd significantly alters both the accuracy (manifested as PSE shifts during electrical microstimulation) and precision (manifested as increases in behavioral threshold after muscimol inactivation) of heading discrimination. Effects were largest during visual heading discrimination and weakest during vestibular heading discrimination. These results suggest that MSTd activity is causally linked to the monkeys' ability to estimate heading during self-motion, although modest effects in some conditions suggest that other areas also contribute to behavior. Despite significant increases in both visual and vestibular discrimination thresholds, the ability to integrate the two cues near-optimally persisted after inactivation of large portions of MSTd bilaterally. Thus, optimal cue integration appears to be a distributed property, such that unaffected brain regions are capable of mediating optimal integration in the absence of a portion of MSTd. Moreover, our simulations suggest that the overall pattern of inactivation results is consistent with contributions of other areas that have vestibulardominant heading tuning.

\section{Optic flow signals in area MSTd}

Area MSTd has long been thought to play an important role in visual heading perception because neurons in this area are selec-
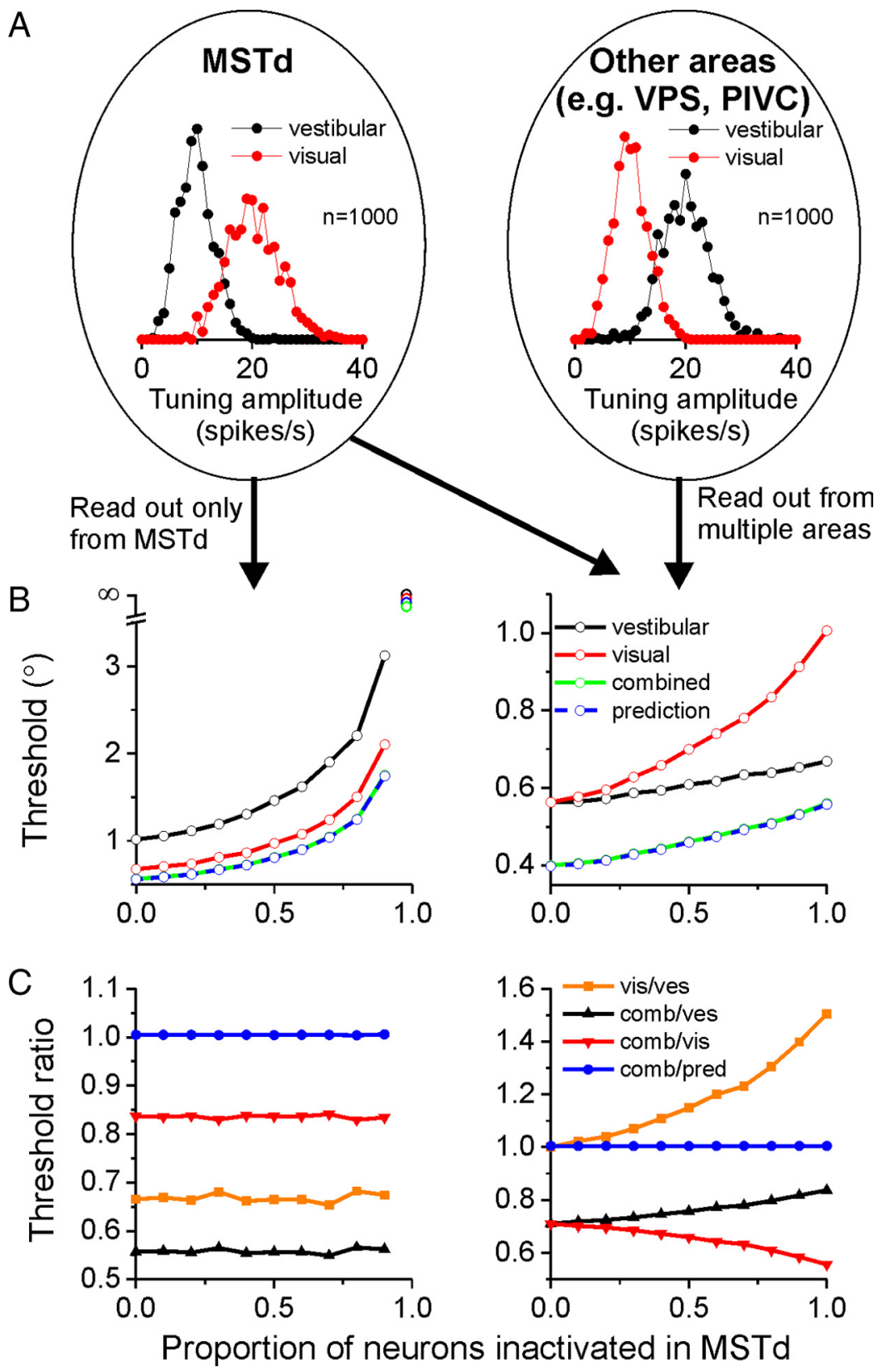

Figure 12. A simple simulation of the effects of inactivation on behavior. Two populations of 1000 hypothetical neurons with cosine heading tuning and uniformly distributed heading preferences were simulated: one population representing area MSTd and the other population representing an area (such as VPS) with vestibular-dominant heading tuning. $\boldsymbol{A}$, Amplitudes of model tuning curves were chosen such that the MSTd-like neurons had heading tuning that was visual dominant (amplitudes were drawn from Poisson distributions with a mean of 20 for visual tuning and a mean of 10 for vestibular tuning). Tuning curves for the VPS-like neurons had mean amplitudes of 10 for visual tuning and 20 for vestibular tuning. A decoder (based on computing population Fisher information) (Gu et al., 2010) pools heading information either from only area MSTd (left column) or from both areas (right column). $\boldsymbol{B}$, Predicted psychophysical thresholds as a function of the proportion of neurons that were inactivated (excluded from decoding) in model area MSTd. C, Same format as in $\boldsymbol{B}$, but instead shows the ratios of predicted thresholds across various stimulus conditions.

tive for complex patterns of optic flow such as those experienced during self-motion (Tanaka et al., 1986, 1989; Duffy and Wurtz, 1991, 1995). A causal link between MSTd activity and visual heading perception has been established previously (Britten and van Wezel, 1998, 2002). Notably, in those studies, predictable effects of microstimulation were mainly seen when animals pursued the fixation target during discrimination. In the absence of pursuit eye movements, observed PSE shifts went in both directions relative to the tuning of MU activity at the stimulation site, 
and the overall effect was not significant (Britten and van Wezel, 1998, 2002). In contrast, we found visual PSE shifts that were predictable from the tuning of MU activity in the vast majority $(86 \%)$ of microstimulation experiments, without pursuit (Fig. $4 A, B)$. Some notable differences in experimental design between the two studies may contribute to this disparity in results. In our study, animals were first trained extensively on the vestibular heading discrimination task before visual cues were introduced gradually in the combined condition, such that animals likely learned to interpret optic flow as indicative of self-motion (Gu et al., 2007, 2008). In contrast, in the studies by Britten and van Wezel $(1998,2002)$, monkeys were trained solely to report the visually specified focus of expansion (FOE). Another potentially important difference involves the configuration of the fixation point and visual stimulus. In our study, monkeys always maintained fixation at the center of the screen, such that the range of visual headings was centered on the fovea. In the studies by Britten and van Wezel $(1998,2002)$, the fixation target was located eccentrically such that the receptive field was brought to the center of the screen and thus the range of headings was centered on the receptive field. For neurons with eccentric receptive fields, the FOE was seldom in the receptive field in our experiments, whereas the FOE was intentionally centered on the receptive field by Britten and van Wezel $(1998,2002)$. Notably, Britten and van Wezel mainly observed predictable microstimulation effects during pursuit conditions, for which the FOE of optic flow would no longer be centered on the MSTd receptive field (similar to our conditions). We speculate that neurons with eccentric receptive fields, which normally experience laminar flow during forward self-motion, may not contribute normally to heading judgments when the FOE is centered on their receptive fields, but this speculation remains to be tested.

A causal role of MSTd in visual heading perception was also supported by our reversible inactivation results. Muscimol, a $\mathrm{GABA}_{\mathrm{A}}$ agonist, suppresses neuronal activity by binding to $\mathrm{GABA}_{\mathrm{A}}$ receptors (Arikan et al., 2002; Edeline et al., 2002; Allen et al., 2008; Liu et al., 2010). Following four injections bilaterally, visual discrimination deficits were large (threefold increase; Fig. $8 A, D$ ) and lasted at least $12 \mathrm{~h}$, in line with previous effects of muscimol at the same concentration (Chowdhury and DeAngelis, 2008). Threshold increases were small, but still significant, following two unilateral or bilateral injections, but were negligible following a single injection (Fig. 9). It is not surprising that large bilateral inactivations of MSTd produced stronger impairments of behavior, considering that MSTd neurons typically have very large receptive fields that span the vertical meridian. Collectively, these results strongly support the hypothesis that optic flow signals in area MSTd are important for heading perception.

\section{Vestibular signals in area MSTd}

Several studies have shown that MSTd neurons are tuned to head/body translation in darkness (Duffy, 1998; Bremmer et al., 1999; Page and Duffy, 2003; Gu et al., 2006). Vestibular responses in MSTd also show trial-to-trial correlations with heading judgments (Gu et al., 2007, 2008). However, we did not observe significant effects of microstimulation on vestibular heading discrimination (Fig. 4C,D). The absence of an effect, however, may simply be due to the relatively weak vestibular tuning of MU responses in MSTd (Fig. 5), which may arise for multiple reasons: (1) fewer $(\sim 60 \%$, compared with $>95 \%)$ neurons are tuned to inertial motion than to optic flow (Duffy, 1998; Page and Duffy, 2003; Gu et al., 2006; Takahashi et al., 2007); (2) even for signif- icantly tuned neurons, the strength of tuning is weaker for vestibular than visual stimuli (Duffy, 1998; Page and Duffy, 2003; Gu et al., 2006; Takahashi et al., 2007); and (3) tuning similarity among nearby MSTd neurons is less consistent for vestibular than visual responses (Chen et al., 2008). Activating neurons with weak or dissimilar tuning properties would generate heterogeneous signals that counteract each other, thus having little or no effect on behavior.

The uncertainty in interpreting microstimulation results for vestibular stimuli is potentially circumvented by using reversible chemical inactivation. Muscimol injections silence regions of cortex much larger than the size of traditional columns such that the outcome should be much less affected by local clustering of neuronal response properties. Indeed, four bilateral muscimol injections significantly elevated vestibular heading thresholds (Fig. $8 B, E$ ), demonstrating that vestibular signals in MSTd do contribute to heading perception. However, even with these large injections, inactivation effects on vestibular heading discrimination were weak $(\sim 20 \%)$ relative to the threefold increases in visual thresholds (Fig. 8, compare $E, D$ ). Our simulations show that this result is expected if other brain areas with stronger vestibular heading tuning also contribute to heading perception. In a series of recent studies, we have shown that there is a range of relative dominance of vestibular and visual heading signals across different areas in the macaque. Specifically, single neurons show a range of dominance from almost pure vestibular tuning in PIVC (Chen et al., 2010), to vestibular-dominant tuning in VPS (Chen et al., 2011b), to relatively balanced visual and vestibular tuning in ventral intraparietal area (VIP) (Chen et al., 2011c), to visualdominant tuning in MSTd (Gu et al., 2006, 2007, 2010), and finally to a complete lack of vestibular signals in MT (Chowdhury et al., 2009). If vestibular-dominant neurons from some of these areas contribute to heading perception, then the pattern of results seen following inactivation of MSTd may be expected based on our simulations (Fig. 12).

\section{Role of MSTd in multisensory heading perception}

We have previously shown that congruent MSTd neurons appear to account for the improvement in behavioral sensitivity that accompanies integration of visual and vestibular heading cues (Gu et al., 2008). Activity of congruent cells also can account for trial-by-trial reweighting of visual and vestibular cues as relative cue reliability varies (Fetsch et al., 2012). One may then wonder how cue integration is affected by partial inactivation of MSTd.

At one extreme, if optimal cue integration involves complex dynamics and precise balancing of network activity across populations of neurons, then one might expect partial inactivation of MSTd to largely destroy the ability to combine cues optimally. However, if optimal cue integration is largely driven by weighted summation of visual and vestibular inputs by single neurons ( $\mathrm{Ma}$ et al., 2006; Fetsch et al., 2012), then optimal cue integration may be quite robust to the loss (or inactivation) of many neurons. Indeed, our simulations (Fig. 12) show that simple decoding of neural populations can yield optimal integration in the face of the loss of a substantial portion of neurons. This is consistent with our findings (Figs. $10,11)$ that monkeys still performed near-optimal integration of visual and vestibular cues following large bilateral inactivations of MSTd that disrupt single-cue thresholds.

In summary, our microstimulation and inactivation results strongly support the idea that MSTd is causally linked to heading 
judgments based on both visual and vestibular signals. Yet, despite significant deficits in single-cue thresholds following bilateral muscimol injections, near-optimal cue integration was not substantially impaired. Our overall pattern of results is consistent with scenarios in which MSTd contributes to heading perception, along with other areas (such as VPS, VIP, and PIVC) that contain representations of self-motion information with a greater dominance of vestibular signals.

\section{References}

Afraz SR, Kiani R, Esteky H (2006) Microstimulation of inferotemporal cortex influences face categorization. Nature 442:692-695.

Allen TA, Narayanan NS, Kholodar-Smith DB, Zhao Y, Laubach M, Brown TH (2008) Imaging the spread of reversible brain inactivations using fluorescent muscimol. J Neurosci Methods 171:30-38.

Angelaki DE, Gu Y, DeAngelis GC (2009) Multisensory integration: psychophysics, neurophysiology, and computation. Curr Opin Neurobiol 19:452-458

Arikan R, Blake NM, Erinjeri JP, Woolsey TA, Giraud L, Highstein SM (2002) A method to measure the effective spread of focally injected muscimol into the central nervous system with electrophysiology and light microscopy. J Neurosci Methods 118:51-57.

Bremmer F, Kubischik M, Pekel M, Lappe M, Hoffmann KP (1999) Linear vestibular self-motion signals in monkey medial superior temporal area. Ann N Y Acad Sci 871:272-281.

Bremmer F, Duhamel JR, Ben Hamed S, Graf W (2002a) Heading encoding in the macaque ventral intraparietal area (VIP). Eur J Neurosci 16:1554-1568.

Bremmer F, Klam F, Duhamel JR, Ben Hamed S, Graf W (2002b) Visualvestibular interactive responses in the macaque ventral intraparietal area (VIP). Eur J Neurosci 16:1569-1586.

Britten KH (1998) Clustering of response selectivity in the medial superior temporal area of extrastriate cortex in the macaque monkey. Vis Neurosci $15: 553-558$

Britten KH (2008) Mechanisms of self-motion perception. Annu Rev Neurosci 31:389-410.

Britten KH, van Wezel RJ (1998) Electrical microstimulation of cortical area MST biases heading perception in monkeys. Nat Neurosci 1: $59-63$.

Britten KH, van Wezel RJ (2002) Area MST and heading perception in macaque monkeys. Cereb Cortex 12:692-701.

Butler JS, Smith ST, Campos JL, Bulthoff HH (2010) Bayesian integration of visual and vestibular signals for heading. J Vis 10:23.

Celebrini S, Newsome WT (1995) Microstimulation of extrastriate area MST influences performance on a direction discrimination task. J Neurophysiol 73:437-448.

Chen A, Gu Y, Takahashi K, Angelaki DE, DeAngelis GC (2008) Clustering of self-motion selectivity and visual response properties in macaque area MSTd. J Neurophysiol 100:2669-2683.

Chen A, DeAngelis GC, Angelaki DE (2010) Macaque parieto-insular vestibular cortex: responses to self-motion and optic flow. J Neurosci 30:3022-3042.

Chen A, DeAngelis GC, Angelaki DE (2011a) A comparison of vestibular spatiotemporal tuning in macaque parietoinsular vestibular cortex, ventral intraparietal area, and medial superior temporal area. J Neurosci 31:3082-3094.

Chen A, DeAngelis GC, Angelaki DE (2011b) Convergence of vestibular and visual self-motion signals in an area of the posterior sylvian fissure. J Neurosci 31:11617-11627.

Chen A, DeAngelis GC, Angelaki DE (2011c) Representation of vestibular and visual cues to self-motion in ventral intraparietal cortex. J Neurosci 31:12036-12052.

Chen LL, Goffart L, Sparks DL (2001) A simple method for constructing microinjectrodes for reversible inactivation in behaving monkeys. J Neurosci Methods 107:81-85.

Chowdhury SA, DeAngelis GC (2008) Fine discrimination training alters the causal contribution of macaque area MT to depth perception. Neuron 60:367-377.
Chowdhury SA, Takahashi K, DeAngelis GC, Angelaki DE (2009) Does the middle temporal area carry vestibular signals related to self-motion? J Neurosci 29:12020-12030.

Cohen MR, Newsome WT (2004) What electrical microstimulation has revealed about the neural basis of cognition. Curr Opin Neurobiol 14:169-177.

Colby CL, Duhamel JR, Goldberg ME (1993) Ventral intraparietal area of the macaque: anatomic location and visual response properties. J Neurophysiol 69:902-914.

Crowell JA, Banks MS (1993) Perceiving heading with different retinal regions and types of optic flow. Percept Psychophys 53:325-337.

de Winkel KN, Weesie J, Werkhoven PJ, Groen EL (2010) Integration of visual and inertial cues in perceived heading of self-motion. J Vis 10:1.

Duffy CJ (1998) MST neurons respond to optic flow and translational movement. J Neurophysiol 80:1816-1827.

Duffy CJ, Wurtz RH (1991) Sensitivity of MST neurons to optic flow stimuli. I. A continuum of response selectivity to large-field stimuli. J Neurophysiol 65:1329-1345.

Duffy CJ, Wurtz RH (1995) Response of monkey MST neurons to optic flow stimuli with shifted centers of motion. J Neurosci 15:5192-5208.

Edeline JM, Hars B, Hennevin E, Cotillon N (2002) Muscimol diffusion after intracerebral microinjections: a reevaluation based on electrophysiological and autoradiographic quantifications. Neurobiol Learn Mem 78:100-124.

Edwards M, O’Mahony S, Ibbotson MR, Kohlhagen S (2010) Vestibular stimulation affects optic-flow sensitivity. Perception 39:1303-1310.

Ernst MO, Banks MS (2002) Humans integrate visual and haptic information in a statistically optimal fashion. Nature 415:429-433.

Fetsch CR, Turner AH, DeAngelis GC, Angelaki DE (2009) Dynamic reweighting of visual and vestibular cues during self-motion perception. J Neurosci 29:15601-15612.

Fetsch CR, DeAngelis GC, Angelaki DE (2010) Visual-vestibular cue integration for heading perception: applications of optimal cue integration theory. Eur J Neurosci 31:1721-1729.

Fetsch CR, Pouget A, DeAngelis GC, Angelaki DE (2011) Neural correlates of reliability-based cue weighting during multisensory integration. Nat Neurosci 15:146-154.

Geesaman BJ, Born RT, Andersen RA and Tootell RB (1997) Maps of complex motion selectivity in the superior temporal cortex of the alert macaque monkey: a double-label 2-deoxyglucose study. Cereb Cortex 7:749-757.

Gu Y, Watkins PV, Angelaki DE, DeAngelis GC (2006) Visual and nonvisual contributions to three-dimensional heading selectivity in the medial superior temporal area. J Neurosci 26:73-85.

Gu Y, DeAngelis GC, Angelaki DE (2007) A functional link between area MSTd and heading perception based on vestibular signals. Nat Neurosci 10:1038-1047.

Gu Y, Angelaki DE, DeAngelis GC (2008) Neural correlates of multisensory cue integration in macaque MSTd. Nat Neurosci 11:1201-1210.

Gu Y, DeAngelis GC, Angelaki DE (2010) Contributions of visual and vestibular signals to 3D heading selectivity in area FEFp. Soc Neurosci Abstr 36:744.

Liu Y, Yttri EA, Snyder LH (2010) Intention and attention: different functional roles for LIPd and LIPv. Nat Neurosci 13:495-500.

Ma WJ, Beck JM, Latham PE, Pouget A (2006) Bayesian inference with probabilistic population codes. Nat Neurosci 9:1432-1438.

Murasugi CM, Salzman CD, Newsome WT (1993) Microstimulation in visual area MT: effects of varying pulse amplitude and frequency. J Neurosci 13:1719-1729.

Nichols MJ, Newsome WT (2002) Middle temporal visual area microstimulation influences veridical judgments of motion direction. J Neurosci 22:9530-9540.

Page WK, Duffy CJ (2003) Heading representation in MST: sensory interactions and population encoding. J Neurophysiol 89:1994-2013.

Royden CS, Banks MS, Crowell JA (1992) The perception of heading during eye movements. Nature 360:583-585.

Salzman CD, Britten KH, Newsome WT (1990) Cortical microstimulation influences perceptual judgements of motion direction. Nature 346:174-177.

Salzman CD, Murasugi CM, Britten KH, Newsome WT (1992) Microstimulation in visual area MT: effects on direction discrimination performance. J Neurosci 12:2331-2355. 
Schlack A, Hoffmann KP, Bremmer F (2002) Interaction of linear vestibular and visual stimulation in the macaque ventral intraparietal area (VIP). Eur J Neurosci 16:1877-1886.

Schlack A, Sterbing-D’Angelo SJ, Hartung K, Hoffmann KP, Bremmer F (2005) Multisensory space representations in the macaque ventral intraparietal area. J Neurosci 25:4616-4625.

Takahashi K, Gu Y, May PJ, Newlands SD, DeAngelis GC, Angelaki DE (2007) Multimodal coding of three-dimensional rotation and translation in area MSTd: comparison of visual and vestibular selectivity. J Neurosci 27:9742-9756.

Tanaka K, Hikosaka K, Saito H, Yukie M, Fukada Y, Iwai E (1986) Analysis of local and wide-field movements in the superior temporal visual areas of the macaque monkey. J Neurosci 6:134-144.
Tanaka K, Fukada Y, Saito HA (1989) Underlying mechanisms of the response specificity of expansion/contraction and rotation cells in the dorsal part of the medial superior temporal area of the macaque monkey. J Neurophysiol 62:642-656.

Uka T, DeAngelis GC (2006) Linking neural representation to function in stereoscopic depth perception: roles of the middle temporal area in coarse versus fine disparity discrimination. J Neurosci 26:6791-6802. van den Berg AV (1992) Robustness of perception of heading from optic flow. Vision Res 32:1285-1296.

van den Berg AV (1996) Judgements of heading. Vision Res 36:2337-2350. Warren WH Jr, Morris MW, Kalish M (1988) Perception of translational heading from optical flow. J Exp Psychol Hum Percept Perform 14:646660 . 